\title{
Aspectos topológicos na teoria geométrica de folheações
}

\author{
Icaro Gonçalves
}

TESE APRESENTADA

$\mathrm{AO}$

Instituto De Matemática e EstatísticA

DA

Universidade De SÃo Paulo

PARA

OBTENÇÃO DO TÍTULO

$\mathrm{DE}$

Doutor EM CIÊNCIAS

Programa: Matemática

Orientador: Prof. Dr. Fabiano Gustavo Braga Brito

Durante o desenvolvimento deste trabalho o autor recebeu auxílio financeiro do CNPq

São Paulo, dezembro de 2016 


\title{
Aspectos topológicos na teoria geométrica de folheações
}

\author{
Esta versão da tese contém as correções e alterações sugeridas \\ pela Comissão Julgadora durante a defesa da versão original do trabalho, \\ realizada em 09/12/2016. Uma cópia da versão original está disponível no \\ Instituto de Matemática e Estatística da Universidade de São Paulo.
}

Comissão Julgadora:

- Prof ${ }^{\mathrm{a}}$. Dr. Fabiano Gustavo Braga Brito (orientador) - IME-USP

- Prof. Dr. Jaime Bruck Ripoll - UFRGS

- Prof. Dr. Marcos Martins Alexandrino da Silva - IME-USP

- Prof. Dr. Dirk Toben - UFSCar

- Prof. Dr. Daniel Miranda Machado - UFABC 
I maintain that mathematics has an object that is just as real as that of geology, particle physics, biochemistry, cosmology, etc, but this object is not material, and it is located in neither space nor time. Nevertheless this object has an existence that is every bit as solid as external reality, and mathematicians bump up against it in somewhat the same way as one bumps into a material object in external reality. Because this reality cannot be located in space or time, it affords - when one is fortunate enough to uncover the minutest portion of it - a sensation of extraordinary pleasure through the feeling of timelessness that it produces - Alain Connes 


\section{Agradecimentos}

Essa é minha singela tentativa de agradecer todos que, de algum modo, fizeram parte dessa longa caminhada.

O apoio incondicional dos meus pais é algo que merece destaque. A simplicidade e o orgulho que vejo em seus olhos é sensação que não tem preço. Sou extremamente grato por tudo que fizeram por mim.

Meu orientador, Fabiano Brito, o agradeço por se dedicar em me ensinar e por me guiar nessa jornada. É uma grande honra tê-lo como mentor nesse rito de passagem. Também sou grato ao Professor Marcos Alexandrino, por diversas conversas e dicas fundamentais para minha carreira.

Agradeço também meus colegas do IME-USP; Benigno, Pablo, Eurípedes. Meus amigos de Minas Gerais; André, Thiago, Guto, Renan, Danyelle, Felipe. Faz uma década que deixei minha cidade e mesmo assim mantivemos viva a nossa amizade.

Meus sinceros agradecimentos aos amigos de São Paulo, com quem pude dividir minhas alegrias, descobertas e momentos de superação. Adriana, que sempre esteve ao meu lado, e que com o passar do tempo, me vi cada vez mais próximo. Alexsandro, que me ajudou a ver a matemática com outros olhos. Allan, por ter me mostrado uma visão profunda sobre as coisas e por se tornar um amigo que nunca imaginei encontrar por aqui. José, por ser um grande amigo e me ensinar sobre matemática, vida e tudo mais. Roberto, por estar sempre ao meu lado e por sua amizade sincera. 


\section{Resumo}

GONÇALVES, I. Aspectos topológicos na teoria geométrica de folheações. 2016. 54 f. Tese (Doutorado) - Instituto de Matemática e Estatística, Universidade de São Paulo, São Paulo, 2016.

Neste trabalho calculamos a classe de Euler de uma folheação umbílica em um ambiente com forma de curvatura apropriada. Combinamos o teorema de Hopf-Milnor e o número de Euler de uma folheação, definido por Connes, para mostrar como a geometria da folheação influencia na topologia da variedade folheada, bem como na topologia da folheação. Além disso, exibimos uma lista de invariantes topológicos para campos vetoriais unitários em hipersuperfícies fechadas do espaço Euclidiano, e mostramos como estes invariantes podem ser empregados como obstruções a certas folheações com geometria prescrita.

Palavras-chave: classe de Euler, folheações umbílicas, grau da aplicação normal de Gauss, hipersuperfícies, campos vetoriais unitários. 


\section{Abstract}

GONÇALVES, I. Topological aspects in the geometric theory of foliations. 2016.

54 p. Tese (Doutorado) - Instituto de Matemática e Estatística, Universidade de São Paulo, São Paulo, 2016.

In this work we compute the Euler class of an umbilic foliation on a manifold with suitable curvature form. We combine the Hopf-Milnor theorem and the Euler number of a foliation, defined by Connes, in order to show how the geometry of the foliation influences the topology of the foliated space as well as the topology of the foliation. Besides, we exhibit a list of topological invariants for unit vector fields on closed Euclidean hypersurfaces, and show how these invariants may be employed as obstructions to certain foliations with prescribed geometry.

Keywords: Euler class, umbilic foliations, degree of the Gauss map, hypersurfaces, unit vector fields. 


\section{Sumário}

Lista de Figuras $\quad$ xi

1 Introdução $\quad 1$

1.1 Objetivos e contribuições . . . . . . . . . . . . . . . . . . . . 4

1.2 Organização do Trabalho . . . . . . . . . . . . . . . . . . 5

$\begin{array}{lll}2 & \text { Preliminares } & 7\end{array}$

2.1 Subvariedades . . . . . . . . . . . . . . . . . . . . . 7

2.2 Curvaturas seccionais de ordem superior . . . . . . . . . . . . . . 11

2.3 Variedades Riemannianas com forma pura de curvatura . . . . . . . . . . . 12

3 A Classe de Euler do fibrado tangente de uma folheação 15

3.1 Classes características . . . . . . . . . . . . . . . . . 15

3.2 Cálculo explícito . . . . . . . . . . . . . . . . . . . . . . . 17

3.3 O caso umbílico e resultados . . . . . . . . . . . . . . . . . 18

3.4 A conjectura de Hopf e o teorema de Milnor . . . . . . . . . . . . . . . . 22

3.4.1 Aplicações do teorema de Milnor . . . . . . . . . . . . . . . . . . . . 22

3.5 Folheações mensuráveis . . . . . . . . . . . . . . . . . . . 24

3.5.1 Número de Euler de uma folheação . . . . . . . . . . . . . . . . . . . 26

3.5.2 Resultados . . . . . . . . . . . . . . . . . . 27

4 Sobre invariantes conformes e construção de exemplos $\quad 29$

4.1 Invariantes conformes . . . . . . . . . . . . . . . . . . 30

4.2 Construção de exemplos na esfera Euclidiana . . . . . . . . . . . . . . . . . 34 
4.3 Folheações umbílicas com fibrado normal integrável . . . . . . . . . . . . . 36

5 Hipersuperfícies Euclidianas: campos de vetores, folheações e o grau da aplicação normal

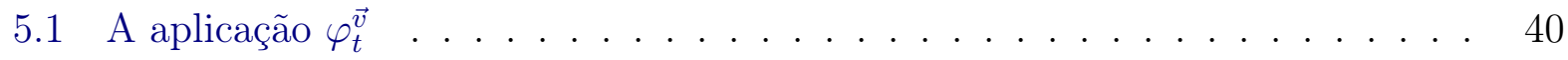

5.2 Invariantes topológicos . . . . . . . . . . . . . . . . . . . . . . 41

5.3 Possíveis valores para o grau da aplicação normal . . . . . . . . . . . . . 44

5.4 Consequências para geometria de folheações . . . . . . . . . . . . . . . . . . 46

5.5 O campo $\vec{v}$ é uma direção principal . . . . . . . . . . . . . . . . . 48

$\begin{array}{ll}\text { Referências Bibliográficas } & 51\end{array}$ 


\section{Lista de Figuras}

3.1 Distribuição com apenas uma folha: plano $x z \ldots \ldots$. . . . . . . . . . 20

4.1 Perturbação dos planos em $\mathbb{R}^{2 n+2} \ldots \ldots \ldots \ldots$. . . . . . . . . . . 34 


\section{Capítulo 1}

\section{Introdução}

A teoria geral de folheações foi estabelecida na década de 40 por Reeb e Ehresmann, e se desenvolveu de maneira rica e extensa nas décadas seguintes. Das ramificações derivadas dos aspectos gerais da teoria, o tratamento de geometria extrínseca de subvariedades merece destaque. Cada folha de uma folheação em uma variedade Riemanniana admite uma estrutura geométrica induzida, e, neste contexto, o seguinte problema surge naturalmente: dada uma variedade $M$ e uma folheação $\mathcal{F}$, existe métrica Riemanniana em $M$, tal que as folhas de $\mathcal{F}$ gozam de estrutura geométrica exigida a priori? Sullivan, em [47], resolveu esta questão de maneira cabal para o caso das folhas serem hipersuperfícies mínimas de $M$,

Teorema 1 (Sullivan, [47]). Seja $M$ uma variedade fechada e $\mathcal{F}$ folheação de codimensão um de $M$. Então $\mathcal{F}$ é minimizável se, e somente se, por toda folha compacta é possivel passar uma curva fechada $\gamma$ transversal à folha e à folheação.

A questão da "geodesibilidade" de uma folheação de codimensão um foi resolvida por Ghys em [22]. A existência de uma métrica que torna as folhas subvariedades totalmente geodésicas é equivalente a $\mathcal{F}$ ser transversal a uma fibração generalizada de Seifert e, além disso, ser difeomorfa a uma folheação modelo, como definida por Ghys também em [22].

Para o caso da existência de uma métrica que torna as folhas umbílicas, Carrière em sua tese, [17], provou, dentre outros resultados, que a folheação de Reeb da esfera $\mathbb{S}^{3}$ pode ser "umbilicalizada", mostrando como a hipótese da umbilicidade é mais fraca perante outras propriedades das folhas, provenientes da segunda forma fundamental. 
Por outro lado, fixada uma propriedade geométrica, indaga-se: quais variedades Riemannianas admitem folheações com características fixadas a priori? quais são as obstruções topológicas, geométricas ou diferenciais para a existência destas folheações?.

Neste quesito, o estudo de folheações umbílicas se destaca nos seguintes trabalhos; $[51,14]$. No primeiro, trata-se de limitantes superiores para a dimensão da folhas e da homogeneidade da folheação, assumindo que a curvatura do ambiente seja positiva, não necessariamente constante. Em ambos os casos, é fundamental que essas folheações possuam a hipótese adicional de serem Riemannianas. Já no segundo artigo, os autores restringem a abordagem para codimensão um em variedades fechadas de dimensão três. Dado que o fibrado normal é integrável, a folheação complementar à umbílica possui um grupo de holonomia que age conforme nas folhas umbílicas, tornando o processo de classificação da folheação de codimensão um totalmente umbílica equivalente à classificação de fluxos holomorfos transversais a folheações de codimensão um.

Em contrapartida ao umbílico, o caso totalmente geodésico possui certas peculiaridades. Para codimensão um, ou mesmo com fibrado normal integrável, existem obstruções geométricas de acordo com curvaturas do ambiente; para o caso das curvaturas seccionais e codimensão arbitrária, veja [12], para codimensão um, e obstruções via outros invariantes geométricos, veja [11] e [26].

Em esferas Euclidianas, uma classificação geométrica de folheações totalmente geodésicas, de codimensões maiores que um, está longe de ser concluída, e isso se ilustra pela abundância de tais folheações. A título de exemplificação, para o caso de codimensão dois em $S^{3}$, existe um teorema na literatura que corrobora com esta afirmação. Denote $\widetilde{G}_{2}\left(\mathbb{R}^{4}\right)$ a variedade Grassmanniana dos 2-planos orientados de $\mathbb{R}^{4}$. Então,

Teorema 2 (Gluck-Warner, [24]). Uma subvariedade de $\widetilde{G}_{2}\left(\mathbb{R}^{4}\right) \cong \mathbb{S}^{2} \times \mathbb{S}^{2}$ corresponde a uma fibração de $S^{3}$ por grandes círculos se e somente se é o gráfico de uma aplicação $f: \mathbb{S}^{2} \rightarrow \mathbb{S}^{2}$ que encurta distância.

Para dimensões maiores e com folhas sendo grandes círculos, pode-se verificar a dificuldade da classificação ainda no nível topológico. Em [25], os autores conjecturam que, 
topologicamente, fibrações de esferas por grandes círculos são topologicamente equivalente a fibrações de Hopf. Nenhuma demonstração completa desta questão foi encontrada até o momento.

Recentemente, Dajczer et al classificaram hipersuperfícies Euclidianas que admitem folheações totalmente geodésicas de codimensão um.

Teorema 3 (Dajczer et al, [21]). Seja $f: M^{n} \rightarrow \mathbb{R}^{n+1}, n \geq 3$, uma imersão isométrica de uma variedade Riemanniana conexa sem pontos flat, munida de uma folheação totalmente geodésica de codimensão um, com folhas completas. Se $f(M)$ não contém uma faixa do tipo superfície, então $f(M)$ ou é uma hipersuperfície regrada ou um tubo parcial sobre uma curva.

O tratamento local foi feito via comparação da imagem do operador forma da hipersuperfície com a distribuição associada à folheação. Ademais, temos uma descrição detalhada de folheações totalmente geodésicas em ambiente de dimensão 4, dada por Cairns e Ghys em [16].

Seja $M^{n+1}(c)$ uma variedade fechada, munida de uma métrica com curvatura seccional constante $c$, e seja $\mathcal{F}$ uma folheação de codimensão um e transversalmente orientada de $M$. Brito et al, em [10], mostraram que as integrais em $M(c)$ das funções simétricas dos autovalores da segunda forma fundamental das folhas, não dependem da folheação,

$$
\int_{M} \sigma_{k}= \begin{cases}c^{k / 2}\left(\begin{array}{c}
n / 2 \\
k / 2
\end{array}\right) \operatorname{vol}(M), & \text { se } k \text { e } n \text { são pares } \\
0, & \text { se } k \text { ou } n \text { é ímpar. }\end{cases}
$$

Para $k=\operatorname{dim}(\mathcal{F})$, o resultado havia sido verificado por Asimov em [2]. Já para $k=1$, a integral se resume a um caso particular de um teorema demonstrado por Reeb em [41], onde se prova que $\int_{M} \sigma_{1}=0$ para qualquer variedade fechada. Como $\sigma_{1}$ é, a menos de uma constante, a função de curvatura média das folhas de $\mathcal{F}$, conclui-se que ou $\sigma_{1}=0$ e todas as folhas são subvariedades mínimas de $M$, ou $\sigma_{1}$ muda de sinal em $M$. Esta observação levou a vários artigos discutindo a problema da "minimalidade" de uma folheação: como caracterizar a existência de uma métrica $M$ que torna as folhas de $\mathcal{F}$ hipersuperfícies mínimas de $M$. Como citado anteriormente, este problema foi completamente resolvido por Sullivan. 
Em ambos os casos, Sullivan e Ghys, a topologia do ambiente e das folhas são peças essenciais na caracterização das propriedades geométricas exigidas das folheações. Além disso, desde os trabalhos de Bott, [5], e Pasternack, [38], relacionando integrabilidade com classes características associadas ao fibrado normal de uma distribuição, diversos resultados surgiram no contexto deste entrelaçamento entre topologia do ambiente folheado e/ou das folhas e as características extrínsecas das folhas, vistas como subvariedades Riemannianas. Diversas classes características se demonstraram ferramentas fundamentais no que diz respeito a este elo. Veja, por exemplo, [6] e [29].

\section{Objetivos e contribuições}

O objetivo central é relacionar a topologia e/ou geometria de uma variedade Riemanniana com a topologia e/ou geometria das folhas de uma folheação.

Especificamente, com alicerce nos trabalhos [8] e [9], essa tese possui as seguintes contribuições:

(a) Construímos uma ponte entre a geometria de folheações umbílicas ou totalmente geodésicas e a topologia do ambiente folheado via a classe de Euler do fibrado tangente à folheação.

(b) Empregamos a demonstração da conjectura de Hopf para dimensão 4 na associação entre folheações umbílicas ou totalmente geodésicas e a classe de Euler.

(c) Combinamos a prova de Milnor da conjectura de Hopf com a definição de Connes do número de Euler de uma folheação, culminando numa relação entre as curvaturas seccionais de uma $S L$-folheação e a média das características de Euler das folhas.

(d) Mostramos uma classe de exemplos de folheações umbílicas que satisfaz as hipóteses dos enunciados supracitados. O método empregado foi a prova de que a propriedade do tensor de curvatura de uma variedade Riemanniana ser diagonalizável é invariante conforme.

(e) Discutimos a ideia de uma folheação umbílica com fibrado normal integrável numa esfera 
Euclidiana. A menos de uma hipótese sobre a 1-forma de curvatura, provamos que tais folheações não existem.

(f) Para hipersuperfícies fechadas do espaço Euclidiano, descobrimos uma lista completamente nova de invariantes topológicos que dependem de um campo unitário e da segunda forma fundamental da hipersuperfície.

(g) Utilizamos os invariantes descobertos para mostrar que o grau da aplicação normal é uma obstrução para folheações cujas folhas possuem certa hipótese sobre a nulidade da segunda forma fundamental.

(h) Finalmente, exibimos alguns invariantes de hipersuperfícies definidos via curvaturas principais.

\section{Organização do Trabalho}

O Capítulo 2 introduz um apanhado de resultados preliminares e trata de assuntos necessários no decorrer da tese: a seção 2.1 fala do método do referencial móvel e subvariedades de ambientes Riemannianos, a 2.2 define as curvaturas de ordem superior, bem como mostra uma relação com a teoria geométrica de folheações. Já a seção 2.3 define e exibe exemplos de variedades com tensor de curvatura diagonalizável.

O Capítulo 3 é uma extensão de [8]. Iniciamos com uma discussão sobre o papel das classes características na teoria de folheações em 3.1 e exibimos um cálculo da classe de Euler em 3.2. As seções 3.3, 3.4 e 3.5 mostram os resultados principais de [8]. A primeira trata do caso de uma folheação umbílica em uma variedade com forma pura de curvatura. Na segunda, a hipótese da pureza é removida, dando lugar às folheações de dimensão 4, com ênfase na demonstração de Milnor da conjectura de Hopf. Finalmente, a terceira seção deste capítulo é voltada as folheações mensuráveis, discussão de um trabalho de Connes e a amálgama deste com o teorema de Milnor-Hopf.

No Capítulo 4, discutimos a invariância conforme da pureza de curvatura na seção 4.1, investigamos uma construção de exemplos de folheações umbílicas em esferas Euclidianas 
na 4.2 e, na seção 4.3, mostramos que não existem folheações umbílicas com fibrado normal integrável nas esferas Euclidianas.

Finalmente, o Capítulo 5 lida com a descoberta de uma lista de invariantes topológicos para hipersuperfícies Euclidianas. A seção 5.1 é voltada para a definição de uma aplicação que mapeia a hipersuperfície numa esfera do Euclidiano. A 5.2 mostra o cálculo explícitos dos invariantes e sua relação com o grau da aplicação normal de Gauss, culminando na seção seguinte, 5.3, onde trabalhamos com um resultado do Milnor que a abundância dos possíveis valores que este grau pode assumir. Concluindo o capítulo, as seções 5.4 e 5.5 tratam, respectivamente, de uma aplicação das fórmulas anteriores para folheações geodésicas e invariantes em função de curvaturas principais da hipersuperfície. 


\section{Capítulo 2}

\section{Preliminares}

\section{Subvariedades}

Neste capítulo preliminar, a notação utilizada e alguns resultados e definições elementares são introduzidos. Para maiores detalhes sobre o método do referencial móvel, indicamos a leitura do livro [37]. Seja $\mathcal{F}$ uma folheação suave da variedade Riemanniana $M$, na qual $\operatorname{dim}(M)=n+p$ e $\operatorname{dim}(\mathcal{F})=n$. Numa vizinhança $U$ de um ponto $x \in M$, tome $\left\{e_{A}\right\}$ referencial ortonormal, tal que o referencial dual, as formas de curvatura e conexão e as equações de estrutura são

$$
\begin{gathered}
\omega_{A}\left(e_{B}\right)=\delta_{A B}, \quad \delta_{A B}=0 \text { se } A \neq B, \quad \delta_{A A}=1 \\
\nabla e_{A}=\sum_{B} \omega_{A B} e_{B}, \quad \omega_{A B}+\omega_{B A}=0 \\
d \omega_{A}=\sum_{B} \omega_{A B} \wedge \omega_{B}, \quad d \omega_{A B}=\sum_{C} \omega_{A C} \wedge \omega_{C B}-\Omega_{A B} \\
\Omega_{A B}=\frac{1}{2} \sum_{C, D} R_{A B C D} \omega_{C} \wedge \omega_{D}, \quad R_{A B C D}+R_{A B D C}=0,
\end{gathered}
$$

onde $1 \leq A, B, C, D, \ldots \leq n+p$. Assumimos que o referencial seja adaptado, no sentido que os primeiros vetores sejam tangentes a $\mathcal{F}$, e os últimos normais. Neste caso, a notação para o conjunto de índices é $1 \leq i, j, \ldots \leq n<\alpha, \beta, \ldots \leq n+p$. Dada uma folha $L$ de $\mathcal{F}$, denotamos por $\theta_{A}$ e $\theta_{A B}$ a restrição de $\omega_{A}$ e $\omega_{A B}$ a $L$. Assim, para qualquer campo $X \in \mathfrak{X} L, \theta_{\alpha}(X)=0$. 
Substituindo nas equações anteriores,

$$
0=d \theta_{\alpha}=\sum_{i} \theta_{\alpha i} \wedge \theta_{i}, \quad d \theta_{i}=\sum_{j} \theta_{i j} \wedge \theta_{j}, \quad \theta_{i j}+\theta_{j i}=0
$$

Pelo lema de Cartan, $\theta_{i \alpha}=\sum_{j} h_{i j}^{\alpha} \theta_{j}$, onde $h_{i j}^{\alpha}=h_{j i}^{\alpha}$. As formas de curvatura de $L$ e $M$ se relacionam pela equação de Gauss,

$$
\Omega_{i j}^{L}=\sum_{\alpha} \theta_{i \alpha} \wedge \theta_{j \alpha}+\Omega_{i j}
$$

Com essa notação, a segunda forma fundamental e o vetor de curvatura média de $L$ são dados respectivamente por

$$
\begin{gathered}
h=\sum_{i, j, \alpha} h_{i j}^{\alpha} \theta_{i} \otimes \theta_{j} \otimes e_{\alpha}, \\
H=\sum_{i, \alpha} h_{i i}^{\alpha} e_{\alpha} .
\end{gathered}
$$

Os fibrados tangente $T L$ e normal $\nu L$ de uma subvariedade $L$ admitem conexões naturalmente associadas com a métrica de $M$. Logo, o fibrado $\otimes^{2} T^{*} L \otimes \nu L$ admite uma única conexão, definida por aquelas provenientes dos fibrados tangente e normal. Como a segunda forma fundamental de $L$ é uma seção de $\otimes^{2} T^{*} L \otimes \nu L$, podemos tomar sua derivada covariante, $\nabla h=\sum h_{i j k}^{\alpha} \theta_{i} \otimes \theta_{j} \otimes \theta_{k} \otimes e_{\alpha}$, com respeito a esta conexão induzida. Os coeficientes $h_{i j k}^{\alpha}$ satisfazem

$$
\sum_{k} h_{i j k}^{\alpha} \theta_{k}=d h_{i j}^{\alpha}+\sum_{l} h_{l j}^{\alpha} \theta_{l i}+\sum_{l} h_{i l}^{\alpha} \theta_{l j}+\sum_{\beta} h_{i j}^{\beta} \theta_{\beta \alpha}
$$

\section{Lema 1.}

$$
\sum_{j, k}\left(h_{i j k}^{\alpha}+\frac{1}{2} R_{\alpha i j k} \theta_{j} \wedge \theta_{k}\right)=0
$$

Demonstração. O caminho da demonstração é simples; tomar a derivada exterior de $\theta_{i \alpha}=$ $\sum_{j} h_{i j}^{\alpha} \theta_{j}$, igualar a equação de estrutura induzida de $L$ e separar os coeficientes de $\nabla h$.

$$
\begin{aligned}
d \theta_{i \alpha} & =\sum_{j}\left(d h_{i j}^{\alpha} \wedge \theta_{j}+h_{i j}^{\alpha} d \theta_{j}\right) \\
& =\sum_{j}\left(d h_{i j}^{\alpha} \wedge \theta_{j}+h_{i j}^{\alpha} \sum_{k} \theta_{j k} \wedge \theta_{k}\right),
\end{aligned}
$$


por outro lado,

$$
\begin{aligned}
d \theta_{i \alpha} & =\sum_{k} \theta_{i k} \wedge \theta_{k \alpha}+\sum_{\beta} \theta_{i \beta} \wedge \theta_{\beta \alpha}-\Omega_{\alpha i} \\
& =\sum_{k, j} h_{k j}^{\alpha} \theta_{i k} \wedge \theta_{j}+\sum_{\beta, j} h_{i j}^{\beta} \theta_{j} \wedge \theta_{\beta \alpha}-\frac{1}{2} \sum_{j, k} R_{\alpha i j k} \theta_{j} \wedge \theta_{k}
\end{aligned}
$$

Igualando ambas,

$$
\sum_{j}\left(d h_{i j}^{\alpha}+\sum_{l} h_{l j}^{\alpha} \theta_{l i}+\sum_{l} h_{i l}^{\alpha} \theta_{l j}+\sum_{\beta} h_{i j}^{\beta} \theta_{\beta \alpha}+\frac{1}{2} \sum_{j, k} R_{\alpha i j k}\right) \wedge \theta_{j}=0
$$

e então obtemos a equação 2.5 .

Uma consequência direta do lema anterior é que, no caso de $M$ ter curvatura seccional constante, $M=M(c)$, os coeficientes de $\nabla h$ satisfazem $h_{i j k}^{\alpha}=h_{i k j}^{\alpha}=h_{j i k}^{\alpha}$, isto é, $h$ é um tensor de Codazzi.

Os resultados anteriores dependem apenas do fato de $L$ ser subvariedade, e não da estrutura de folheação introduzida. Suponha, agora, que $L$ seja uma hipersuperfície de $M(c)$, não necessariamente folha de uma folheação. Estamos interessados na estrutura geométrica extrínseca de $L$. Defina $\Gamma_{i j}^{k}=\theta_{i j}\left(e_{k}\right)$, funções que, de acordo com a notação definida anteriormente, são formas de curvatura restritas a $L$. Podemos fixar uma direção normal e calcular, em termos dos $\Gamma_{i j}^{k}$, os coeficientes $h_{i j k}$.

Proposição 1. Em cada ponto de L, vale

$$
h_{i j k}=\delta_{i j} d\left(\lambda_{i}\right)\left(e_{k}\right)+\left(\lambda_{i}-\lambda_{j}\right) \Gamma_{i j}^{k}
$$

Demonstração. Pela relação (2.4),

$$
\begin{aligned}
h_{i j k} & =\sum_{k} h_{i j k} \theta_{k}\left(e_{k}\right) \\
& =d h_{i j}\left(e_{k}\right)+\sum_{l} h_{l j} \theta_{l i}\left(e_{k}\right)+\sum_{l} h_{i l} \theta_{l j}\left(e_{k}\right) \\
& =d h_{i j}\left(e_{k}\right)+\sum_{l} h_{l j} \Gamma_{l i}^{k}+\sum_{l} h_{i l} \Gamma_{l j}^{k}
\end{aligned}
$$


Supor que a matriz da segunda forma de $L,\left(h_{i j}\right)$, é diagonalizada no ponto $p \in L$. Deste modo, neste ponto, $\left(h_{i j}\right)=\delta_{i j} \lambda_{i}$, donde segue-se a tese.

Comparadas aos coeficientes de estrutura dados pelo colchete de Lie $\left[e_{i}, e_{j}\right]=\sum_{k} C_{i j k} e_{k}$, as funções $\Gamma_{i j}^{k}$ se relacionam como,

$$
C_{i j k}=\Gamma_{j k}^{i}-\Gamma_{i k}^{j}
$$

Isso se deve ao fato de que a conexão induzida em $L$ é conexão de Levi-Civita, ie compatível com a métrica.

Cada curvatura principal $\lambda_{i}$ de $L$ define uma distribuição $\mathcal{D}_{i}$, dada pelas direções principais associadas a $\lambda_{i}$. Uma questão que surge naturalmente é o problema da integrabilidade desta distribuição.

Teorema 4 (Ôtsuki, [36]). Seja $M$ uma hipersuperfície em uma variedade Riemanniana de curvatura seccional constante, tal que as multiplicidades das curvaturas principais $\lambda_{i}$ são constantes. Então as distribuições $\mathcal{D}_{i}$ são integráveis. Se a multiplicidade de uma curvatura principal é maior que um, então esta curvatura é constante ao longo das folhas correspondentes a folheação associada a distribuição de seus autovetores.

Como subvariedades de $L$, a geometria extrínseca nas folhas associadas as curvaturas principais é naturalmente derivada de $L$, no sentido que o operador forma destas folhas depende apenas das curvaturas de $L$. Especificamente,

Teorema 5. Seja $\gamma$ uma folha associada ao autovalor $\lambda$ e tome $T \gamma$ como o espaço tangente de $\gamma$. Seja $\mu \neq \lambda$ curvatura principal de L, com $N$ autovetor associado. Então o operador forma de $\gamma$ na direção $N, A_{N}: T \gamma \rightarrow T \gamma$ é dado por

$$
A_{N}(X)=\frac{d \lambda(N)}{\lambda-\mu} X
$$

Demonstração. A prova deste resultado pode ser encontrada no livro Geometry of Hypersurfaces, de Cecil e Ryan, [18]; o caso de multiplicidade maior que um está na demonstração 
do teorema 2.11, páginas 25 e 26, e para multiplicidade igual a um, no teorema 2.18, página 31.

Na sequência, introduzimos dois tipos importantes de subvariedades, que serão úteis nos capítulos onde exibimos os resultados principais.

Definição 1. Uma subvariedade imersa $L$ de $M$ é dita umbilica quando $h(\cdot, \cdot)=\langle\cdot, \cdot\rangle H$.

Intuitivamente, umbilicidade significa que a subvariedade se curva da mesma maneira para qualquer direção tangente, bem como a esfera Euclidiana, que é o exemplo que melhor traduz essa propriedade. Dizemos que a subvariedade $L$ é totalmente geodésica no caso em que for simultaneamente umbílica e $H$ identicamente nulo. Neste caso, toda geodésica em $L$ também é uma geodésica da variedade ambiente. Exemplos imediatos consistem de hiperplanos no espaço Euclidiano, grandes círculos em esferas Euclidianas e curvas geodésicas em variedades quaisquer.

\section{Curvaturas seccionais de ordem superior}

O objetivo desta seção é introduzir um conceito geométrico que estende a noção de curvatura seccional de um ambiente Riemanniano. Seguindo [48],

Definição 2. Seja M uma variedade Riemanniana e q um inteiro positivo par. Se $P$ é um q-plano orientado de $M$, então definimos a q-ésima curvatura seccional $\gamma_{q}(P)$ de $M$ como

$$
\gamma_{q}(P)=\frac{1}{q !} \sum_{\sigma \in \mathcal{S}_{q}} \operatorname{sgn}(\sigma) \Omega_{\sigma(1) \sigma(2)} \wedge \cdots \wedge \Omega_{\sigma(q-1) \sigma(q)}\left(e_{1}, \ldots, e_{q}\right)
$$

onde $\left\{e_{1}, \ldots, e_{q}\right\}$ é uma base ortonormal de $P$.

Comparadas à curvatura seccional usual, que neste caso se reduz à $\gamma_{2}$, curvaturas seccionais de ordem superior são invariantes mais fracos da estrutura geométrica de $M$. Isso se deve ao fato que, se em uma variedade Riemanniana compacta orientada $M$ a $q$-ésima curvatura seccional $\gamma_{q}$ é constante, então a $k$-ésica classe de Pontryagin de $M$ se anula, para $k \geq q / 2$, veja Teorema A de [48]. Além disso, as curvaturas $\gamma_{q}$ medem a curvatura de Lipschitz-Killing de subvariedades geodésicas $q$-dimensionais de $M$. O caso mais simples é quando $M$ possui 
curvatura seccional constante $c$; as curvaturas de ordem superior são apenas potências de $c$. Se $M$ e $N$ são variedades Riemanninas. com $N$ flat, então $\gamma_{q}=0$, para todo $q \geq \operatorname{dim}(N)$. Completando a lista de exemplos, vale mencionar que, se uma variedade Riemanniana $M^{n}$ é homogênea, então $\gamma_{n}$ é constante (para mais detalhes, vide [48]).

No que diz respeito a teoria geométrica de folheações, em [11], Brito exibiu uma obstrução a folheações totalmente geodésicas via curvaturas seccionais de ordem superior. Especificamente, provou o seguinte resultado

Teorema 6 (Brito, [11]). Se existe um inteiro par, $2 \leq q \leq n$, tal que as curvaturas $q$ seccionais de uma variedade $M^{n}$ são não nulas, então $M$ não admite folheação totalmente geodésica de codimensão um.

Em particular, variedades com curvatura seccional constante diferente de zero não admitem tais folheações.

\section{Variedades Riemannianas com forma pura de curvatura}

Se, por um lado, podemos estender a noção de curvatura seccional para invariantes mais primitivos, podemos procurar por variedades nas quais a forma de curvatura $\Omega_{A B}$ seja escrita em termos simples. Olhando para aquelas com curvatura seccional constante, tem-se simplesmente $\Omega_{A B}=c \omega_{A} \wedge \omega_{B}$. Uma vez que a constante $c$ aparece para qualquer referencial ortonormal, podemos considerar uma generalização direta, aquela na qual considera a existência de um referencial que diagonaliza o tensor de curvatura e nos garante a simplicidade desejada. Especificamente, seguimos [4, 39] e definimos

Definição 3. Uma variedade Riemanniana $M$ é dita possuir forma de curvatura pura sempre que existir um referencial $\left\{e_{A}\right\}$, tal que $\Omega_{A B}=c_{A B} \omega_{A} \wedge \omega_{B}$, em todo ponto de $M$, onde $c_{A B}$ é a curvatura seccional do plano gerado por $e_{A}$ e $e_{B}$.

Existem diversas classes de ambientes Riemannianos que satisfazem a condição da definição acima.

Exemplo 1. Seja $L^{n}$ uma hipersuperfície imersa em uma forma espacial $M^{n+1}(c)$. Pela equação (2.1), $\Omega_{i j}^{L}=\omega_{i n+1} \wedge \omega_{j n+1}+c \omega_{i} \wedge \omega_{j}$. Dado um ponto $x \in L$, seja $\left\{e_{i}\right\}$ um referencial 
que diagonaliza a segunda forma fundamental de $L$ em uma vizinhança de $x$. Logo, $\omega_{i n+1}=$ $\lambda_{i} \omega_{i}$, com $\lambda_{i}$ função contínua definida na vizinhança de $x$; curvatura principal de L. Assim $\Omega_{i j}^{L}=\left(\lambda_{i} \lambda_{j}+c\right) \omega_{i} \wedge \omega_{j}$.

Exemplo 2. Mais geralmente, seja $L^{n}$ uma variedade imersa em uma forma espacial $M^{n+p}(c)$, tal que o fibrado normal seja plano. Neste caso, é imediato verificar que as matrizes $\left(h_{i j}^{\alpha}\right)$ e $\left(h_{i j}^{\beta}\right)$ podem ser diagonalizadas simultaneamente, para ambos vetores normais $e_{\alpha}$ e $e_{\beta}$. Assim, pela equação (2.1), temos que L possui forma de curvatura pura.

Exemplo 3. Fixe $M^{n}$ uma variedade Riemanniana. De acordo com [39], página 92, podemos usar o produto de Kulkarni-Nomizu, denotado por o, para escrever o tensor de curvatura em termos da curvatura escalar, curvatura de Ricci e tensor de Weyl. Quando $n \geq 4$,

$$
R=\frac{\text { scal }}{2 n(n-1)}\langle\cdot, \cdot\rangle \circ\langle\cdot, \cdot\rangle+\left(\operatorname{Ric}-\frac{\mathrm{scal}}{n}\langle\cdot, \cdot\rangle\right) \circ\langle\cdot, \cdot\rangle+W
$$

e para $n=3$,

$$
R=\frac{\mathrm{scal}}{12}\langle\cdot, \cdot\rangle \circ\langle\cdot, \cdot\rangle+\left(\operatorname{Ric}-\frac{\mathrm{scal}}{3}\langle\cdot, \cdot\rangle\right) \circ\langle\cdot, \cdot\rangle
$$

Desse modo, se $\operatorname{dim} M=3$ ou $W=0$, então $M$ possui forma de curvatura pura. No segundo caso, também enunciado em [39], M possui tensor de Weyl nulo se e somente se sua métrica for localmente conforme à métrica plana.

Segue diretamente da definição que, se $M$ e $N$ possuem forma pura de curvatura, então o produto Riemanniano $M \times N$ também o possui. A recíproca deste fato é verdadeira, e foi demonstrada em [35].

Admitir métrica com forma de curvatura tendo tal propriedade implica em consequências para a topologia da variedade,

Teorema 7 (Noronha, [35]). Se M admite uma métrica com forma pura de curvatura, então todas as classes de Pontrjagin de $M$ se anulam. 


\section{Capítulo 3}

\section{A Classe de Euler do fibrado tangente de uma folheação}

\section{Classes características}

Classes características são classes cohomológicas associadas a fibrados vetoriais, definidas no espaço base, as quais medem a maneira que as fibras destes fibrados se torcem sobre a base. Considerando aplicações à teoria de folheações, os primórdios datam de um teorema de Milnor e Wood, que tratam da classe de Euler como uma obstrução à existência de folheações transversas as fibras de fibrados circulares torcidos ${ }^{1}$, sobre superfícies. Acerca de obstruções a integrabilidade, destaca-se o teorema de Bott, no qual diz que uma distribuição de codimensão $p$ no fibrado tangente ser integrável, implica que classes de Pontryagin associadas ao fibrado normal da folheação se anulam para graus acima de $2 p$. Posteriormente, Pasternack otimiza tal resultado para distribuições tangentes a folheações Riemannianas, com anulamento dessas classes a partir do grau $p$.

Folheações são subfibrados integráveis do fibrado tangente, e, nos moldes dos resultados anteriores, pode-se destacar restrições topológicas quando as classes tratadas são relacionadas as distribuições associadas as folheações em si. Neste contexto, trata-se não somente os cálculos das classes, mas relações com propriedades geométricas impostas a priori as folheações.

Por exemplo, quando a folheação é totalmente geodésica, temos

\footnotetext{
${ }^{1}$ Do inglês overly twisted circle bundles.
} 
Teorema 8 (Johnson-Naveira, [29]). Seja $\mathcal{F}$ uma folheação totalmente geodésica, $\operatorname{dim}(\mathcal{F})=$ $n$ impar, de uma variedade Riemanniana $(n+p)$-dimensional $M$. Então a $(n+p)$-ésima classe de Pontryagin de $\mathcal{F}$ se anula.

Para folheações por superfícies, com fibrado normal integrável a uma folheação mínima, temos

Teorema 9 (Brito, [6]). Seja $M^{n+2}$ uma variedade Riemanniana orientada e fechada e $\mathcal{F}_{1}$ uma folheação minima de classe $C^{\infty}$ de $M, \operatorname{dim}\left(\mathcal{F}_{1}\right)=n$. Suponha que o fibrado normal de $\mathcal{F}_{1}$, digamos $\mathcal{F}_{2}$, seja $C^{\infty}$ e integrável e que ambas $\mathcal{F}_{1}$ e $\mathcal{F}_{2}$ sejam orientáveis.

1. Se $\operatorname{Ric}(M)>0$, então $\epsilon\left(\mathcal{F}_{2}\right) \neq 0$.

2. Se $\operatorname{Ric}(M) \geq 0$, então ou $\mathcal{F}_{1}$ é totalmente geodésica ou $\epsilon\left(\mathcal{F}_{2}\right) \neq 0$ (ambos podem acontecer simultaneamente).

3. Se $M$ tem curvatura seccional não-negativa, então ou $\epsilon\left(\mathcal{F}_{2}\right) \neq 0$ ou $\mathcal{F}_{1}$ e $\mathcal{F}_{2}$ são totalmente geodésicas (ambos podem acontecer simultaneamente).

Onde $\operatorname{Ric}(M)$ denota a curvatura de Ricci de $M$.

Na sequência, definiremos a classe de Euler associada ao fibrado tangente de uma folheação. Suponha $n=2 k$. Seja $H^{*}(M, \mathbb{R})$ a cohomologia de de Rham de $M^{n+p}$, e denote por $\mathcal{D}$ o fibrado tangente de $\mathcal{F}^{n}$. Considerando o referencial e as formas de curvatura consideradas anteriormente e, de acordo com [31], página $318, \epsilon(\mathcal{D}) \in H^{2 k}(M, \mathbb{R})$ é escrita como

$$
\epsilon(\mathcal{D})=\frac{(-1)^{k}}{(4 \pi)^{k} k !} \sum_{\sigma \in \mathfrak{S}_{2 k}} \operatorname{sgn}(\sigma) \Omega_{\sigma(1) \sigma(2)}^{\mathcal{F}} \wedge \cdots \wedge \Omega_{\sigma(2 k-1) \sigma(2 k)}^{\mathcal{F}}
$$

A equação (2.1) é a maneira natural de escrever as curvaturas das folhas em temos da segunda forma fundamental e das curvaturas do ambiente,

$$
\begin{aligned}
\epsilon(\mathcal{D}) & =\frac{(-1)^{k}}{(4 \pi)^{k} k !} \sum_{\sigma \in \mathfrak{S}_{2 k}} \operatorname{sgn}(\sigma)\left(\sum_{\alpha} \theta_{\sigma(1) \alpha} \wedge \theta_{\sigma(2) \alpha}+\Omega_{\sigma(1) \sigma(2)}\right) \wedge \cdots \\
& \cdots \wedge\left(\sum_{\alpha} \theta_{\sigma(2 k-1) \alpha} \wedge \theta_{\sigma(2 k) \alpha}+\Omega_{\sigma(2 k-1) \sigma(2 k)}\right) .
\end{aligned}
$$


Vale salientar que, diferentemente das classes de Chern e Pontryagin, que podem ser obtidas via quaisquer conexão definidas no fibrado em questão, a classe de Euler pode ser computada somente via conexão compatível com a métrica (para mais detalhes, ver [31]).

Se $M$ é uma variedade compacta orientada de dimensão par, e $\mathcal{D}=T M$ é o fibrado tangente de $M$, então a classe de Euler $\epsilon(\mathcal{D})$ integrada em $M$ devolve a característica de Euler de $M$. Este é o teorema de Gauss-Bonnet-Chern, [19].

\section{Cálculo explícito}

Primeiramente, vejamos como escrever a classe de Euler de uma folheação, com uma hipótese adicional sobre o fibrado normal. Seja $L$ uma folha da folheação $\mathcal{F}^{2 k}$, que possua fibrado normal plano. A segunda forma fundamental de $L$ pode ser diagonalizada simultaneamente em qualquer direção normal; localmente denotada por $e_{\alpha}$. Logo, $\theta_{i \alpha}=\lambda_{i}^{\alpha} \theta_{i}$, e então

$$
\begin{aligned}
\epsilon(\mathcal{D})= & \frac{(-1)^{k}}{(4 \pi)^{k} k !} \sum_{\sigma \in \mathfrak{S}_{2 k}} \operatorname{sgn}(\sigma)\left(\sum_{\alpha} \lambda_{\sigma(1)}^{\alpha} \lambda_{\sigma(2)}^{\alpha} \theta_{\sigma(1)} \wedge \theta_{\sigma(2)}+\Omega_{\sigma(1) \sigma(2)}\right) \wedge \cdots \\
\cdots & \wedge\left(\sum_{\alpha} \lambda_{\sigma(2 k-1)}^{\alpha} \lambda_{\sigma(2 k)}^{\alpha} \theta_{\sigma(2 k-1)} \wedge \theta_{\sigma(2 k)}+\Omega_{\sigma(2 k-1) \sigma(2 k)}\right) \\
= & \frac{(-1)^{k}}{(4 \pi)^{k} k !} \sum_{l=0}^{k}\left(\begin{array}{c}
k \\
l
\end{array}\right) \sum_{\sigma \in \mathfrak{S}_{2 k}} \operatorname{sgn}(\sigma)\left(\sum_{\alpha_{1}} \lambda_{\sigma(1)}^{\alpha_{1}} \lambda_{\sigma(2)}^{\alpha_{1}}\right) \cdots\left(\sum_{\alpha_{l}} \lambda_{\sigma(2 l-1)}^{\alpha_{l}} \lambda_{\sigma(2 l)}^{\alpha_{l}}\right) \\
& \theta_{\sigma(1)} \wedge \cdots \wedge \theta_{\sigma(2 l)} \wedge \Omega_{\sigma(2 l+1) \sigma(2 l+2)} \wedge \cdots \wedge \Omega_{\sigma(2 k-1) \sigma(2 k)}
\end{aligned}
$$

Assumindo $\mathcal{F}$ folheação totalmente umbílica, o campo de curvatura média $H$ se escreve como $H=\sum_{\alpha} \lambda_{\alpha} e_{\alpha}$, onde $\lambda_{\alpha}$ é a curvatura principal na direção $e_{\alpha}$; basta usar o fato de que $h_{i i}^{\alpha}=\left\langle H, e_{\alpha}\right\rangle$. Assim, $\theta_{i \alpha}=\lambda_{\alpha} \theta_{i}$, donde escrevemos a classe de Euler de uma folheação umbílica como

$\epsilon(\mathcal{F})=\frac{(-1)^{k}}{(4 \pi)^{k} k !} \sum_{l=0}^{k}\left(\begin{array}{l}k \\ l\end{array}\right)\|H\|^{2 l} \sum_{\sigma \in \mathfrak{S}_{2 k}} \operatorname{sgn}(\sigma) \theta_{\sigma(1)} \wedge \cdots \wedge \theta_{\sigma(2 l)} \wedge \Omega_{\sigma(2 l+1) \sigma(2 l+2)} \wedge \cdots \wedge \Omega_{\sigma(2 k-1) \sigma(2 k)}$

onde $\|H\|^{2}=\sum_{\alpha} \lambda_{\alpha}^{2}$ e o parâmetro $l$ indica a quantidade de fatores $\Omega_{i j}$ e $\lambda_{\alpha}^{2} \theta_{i} \wedge \theta_{j}$ que aparecem quando o produto exterior é distribuído perante a soma. Como a soma corre em 
termos de permutações em $\mathfrak{S}_{2 k}$, podemos verificar a repetição de termos, e agrupá-los a menos de um fator combinatório $\left(\begin{array}{l}k \\ l\end{array}\right)$.

Em termos da classe de variedades introduzida anteriormente, a classe de Euler de $\mathcal{F}$ admite uma apresentação simplificada,

Lema 2. Se $\mathcal{F}$ é umbílica e $M$ possui forma pura de curvatura, então

$$
\epsilon(\mathcal{D})=\frac{(-1)^{k}}{(4 \pi)^{k} k !} \sum_{l=0}^{k}\left(\begin{array}{l}
k \\
l
\end{array}\right)(2 l) !(2 k-2 l) !|| H \|^{2 l} \gamma_{2 k-2 l} \theta_{1} \wedge \cdots \wedge \theta_{2 k}
$$

onde $\gamma_{p}$ é a p-ésima curvatura seccional de $M$, como em (2.8).

Demonstração. Com a hipótese de forma pura de curvatura, a equação (3.3) é escrita como,

$$
\begin{aligned}
\epsilon(\mathcal{F})= & \frac{(-1)^{k}}{(4 \pi)^{k} k !} \sum_{l=0}^{k}\left(\begin{array}{l}
k \\
l
\end{array}\right)\|H\|^{2 l} \sum_{\sigma \in \mathfrak{S}_{2 k}} \operatorname{sgn}(\sigma) \theta_{\sigma(1)} \wedge \cdots \wedge \theta_{\sigma(2 l)} \wedge \\
& c_{\sigma(2 l+1) \sigma(2 l+2)} \theta_{\sigma(2 l+1)} \wedge \theta_{\sigma(2 l+2)} \wedge \cdots \wedge c_{\sigma(2 k-1) \sigma(2 k)} \theta_{\sigma(2 k-1)} \wedge \theta_{\sigma(2 k)}
\end{aligned}
$$

Usando as propriedades do grupo de permutação, agrupamos as curvaturas seccionais $c_{i j}$ do seguinte modo,

$$
\epsilon(\mathcal{D})=\frac{(-1)^{k}}{(4 \pi)^{k} k !} \sum_{l=0}^{k}\left(\begin{array}{l}
k \\
l
\end{array}\right)\|H\|^{2 l} \sum_{\sigma \in \mathfrak{S}_{2 k}} \operatorname{sgn}(\sigma) c_{\sigma(1) \sigma(2)} \cdots c_{\sigma(2 k-2 l-1) \sigma(2 k-2 l)} \theta_{\sigma(1)} \wedge \cdots \wedge \theta_{\sigma(2 k)}
$$

Usando o fato de $c_{i j}$ ser simétrica, obtemos a equação desejada.

\section{O caso umbílico e resultados}

Com a notação e ambiente folheado fixados, podemos enunciar o primeiro resultado,

Teorema 10 (Brito-Gonçalves, [8]). Seja $\mathcal{D}^{2 k}$ uma distribuição em uma variedade Riemanniana $M^{2 k+p}$ com forma de curvatura pura. Seja L uma subvariedade fechada, i.e. compacta e sem bordo, umbílica (totalmente geodésica) de $M$, com dimensão $2 k$, e suponha que as curvaturas seccionais de $M$, restritas a $L$, sejam não-negativas (positivas). Se $\mathcal{D}$ é tangente a L, então $\epsilon(\mathcal{D}) \neq 0$. 
Demonstração. A imersão $i: L \rightarrow M$ induz naturalmente $i^{*}: H^{2 k}(M, \mathbb{R}) \rightarrow H^{2 k}(L, \mathbb{R})$ e temos diretamente a integral $\int_{L} i^{*}(\epsilon(\mathcal{D}))$. Além disso, $L$ é subvariedade umbílica, donde a equação (3.4) é satisfeita. Suponha, por absurdo, que $\epsilon(\mathcal{D})$ seja uma $2 k$-forma exata. Logo, a integral anterior é zero pelo teorema de Stokes, e o fato de termos uma integral de funções positivas, em termos da norma da curvatura média de $L$ e das curvaturas seccionais de $M$, implica em uma contradição. Portanto, $\epsilon(\mathcal{D}) \neq 0$ in $H^{2 k}(M, \mathbb{R})$.

Devemos destacar que não é necessária nenhuma hipótese sobre a completude da métrica de $M$, apenas a propriedade de admitir um referencial que diagonaliza seu tensor de curvatura.

O teorema 10 trata de distribuições que são tangentes a uma subvariedade, com mesma dimensão. A título de ilustração, poderíamos pensar numa distribuição com apenas uma folha, como na figura (3.1). Em cada reta $r$ paralela ao eixo $x$, define-se uma distribuição de planos onde cada plano contém $r$. Ainda, a inclinação destes planos em relação com o plano $x z$, depende da posição na reta $r$. Quanto mais longe de $x z$, mais rapidamente os planos giram em torno da reta que os define. Em xz, onde não há inclinação alguma, a distribuição é dada por $p \mapsto x z$, donde é integrável apenas nas retas paralelas ao eixo $x$ e pertencentes a $x z$.

A seguir listamos algumas consequências do teorema 10. Os corolários abrangem desde resultados de rigidez até consequências para folheações em variedades Riemannianas fixadas.

Corolário 1. Seja $M^{2 k+p}(c)$ uma variedade Riemanniana de curvatura constante $c \geq 0$, munida de uma distribuição $\mathcal{D}^{2 k}$. Assuma que $\mathcal{D}$ seja tangente (ou possua uma folha) a uma subvariedade compacta L. Se $H^{2 k}(M)=0$ e L é umbílica, então c $=0$ e L é totalmente geodésica.

Demonstração. Neste caso, a equação (2.1) é escrita como $\Omega_{i j}^{L}=\left(\|H\|^{2}+c\right) \theta_{i} \wedge \theta_{j}$, e

$$
\epsilon(\mathcal{D})=\frac{(-1)^{k}(2 k) !}{(4 \pi)^{k} k !} \sum_{l=0}^{k}\left(\begin{array}{l}
k \\
l
\end{array}\right)\|H\|^{2 l} c^{k-l} \theta_{1} \wedge \cdots \wedge \theta_{2 k}
$$

Logo, $H^{2 k}(M)=0$ implica que $\epsilon(D)=0$, donde a integral em $L$ resulta na tese. 


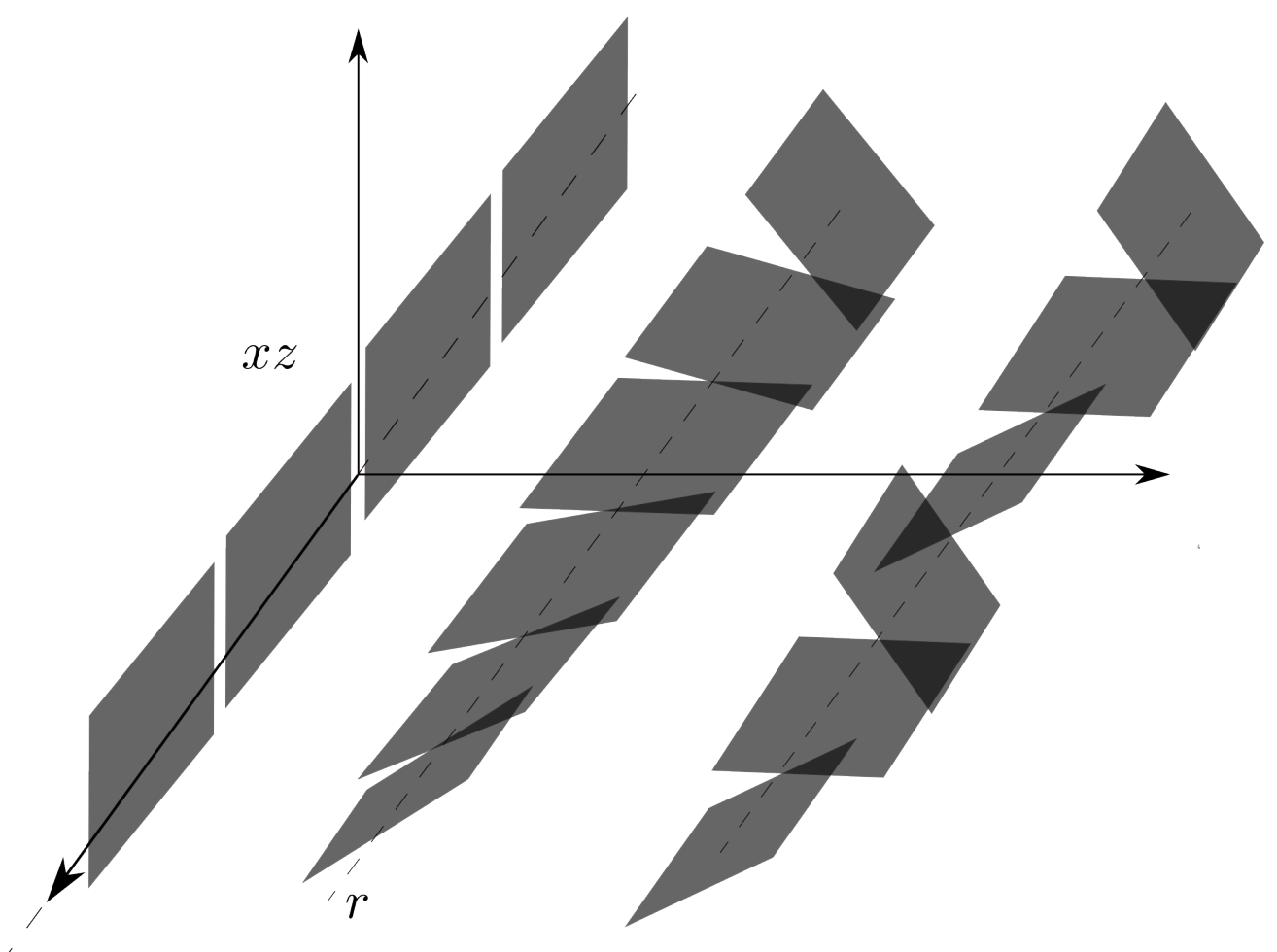

Figura 3.1: Distribuição com apenas uma folha: plano $x z$

Corolário 2. Seja $M^{2 k+p}$ uma esfera homológica imersa no espaço Euclidiano. Se as curvaturas seccionais de $M$ são nãonegativas (positivas) ao longo de uma certa subvariedade umbílica (totalmente geodésica) $L^{2 k}$ de $M$, então $M$ não admite distribuição $\mathcal{D}^{2 k}$ tangente a $L$.

Demonstração. O fato de ser uma esfera homológica implica $H^{2 k}(M)=0$.

Veremos, no último capítulo desta tese, que no caso de codimensão um e folheação totalmente geodésica, podemos remover a hipótese sobre as curvaturas seccionais das esferas homológicas.

Corolário 3. Esferas Euclidianas não admitem folheação com codimensão impar e pelo menos uma folha fechada umbilica.

Demonstração. Esferas de dimensão par não admitem nenhum tipo de folheação regular, logo, a dimensão da esfera deve ser ímpar. Com efeito, caso a folheação do enunciado tenha uma folha fechada umbílica, usamos os resultados anteriores e concluímos que a classe de Euler desta folheação é não nula, donde chegamos em uma contradição. 
Finalmente, alguns comentários sobre a importância das hipóteses no teorema 10. Primeiramente, pede-se, no caso da subvariedade ser totalmente geodésica, que as curvaturas seccionais ao longo dessa folha sejam positivas. Isso se deve diretamente ao fato de como a classe de Euler se escreve quando restringimos o tratamento a este caso. Na equação (3.4), o caso totalmente geodésico elimina todos os termos dependentes do campo de curvatura média, exceto o termo que possui o produto de todas curvaturas seccionais.

$$
\epsilon(\mathcal{D})=\frac{(-1)^{k}}{(4 \pi)^{k} k !}(2 k) ! \gamma_{2 k} \theta_{1} \wedge \cdots \wedge \theta_{2 k}
$$

Isso significa que, se pelo menos uma curvatura é nula, então este último termo também se anula, donde $\epsilon(\mathcal{D})=0$.

Por outro lado, podemos construir uma folheação umbílica, com dimensão par, tal que sua classe de Euler seja nula. Tome $\mathcal{F}$ folheação por grandes círculos de $\mathbb{S}^{3}$. No produto $\mathbb{S}^{3} \times \mathbb{S}^{3}$, a folheação produto $\mathcal{F} \times \mathcal{F}$ é geodésica, tem dimensão par e todas as folhas são compactas. Agora, tomando uma função suave, constante ao longo das folhas, e fazendo uma mudança conforme na métrica canônica de $\mathbb{S}^{3} \times \mathbb{S}^{3}$, temos como produto final uma folheação umbílica, com dimensão par e todas as folhas são compactas. Já que $H^{2}\left(\mathbb{S}^{3}\right)=0$, então a classe de Euler de $\mathcal{F} \times \mathcal{F}$ é nula. Ainda, pode-se deduzir diretamente, sem saber calcular $H^{2}\left(\mathbb{S}^{3}\right)$, que esta classe é nula. Porém, tais cálculos dependem de equações para mudança conforme de métricas, que é o tema do capítulo seguinte. 


\section{A conjectura de Hopf e o teorema de Milnor}

A partir do momento que a hipótese da diagonalização do tensor de curvatura é introduzida, a classe de Euler se torna computável, no sentido de termos uma fórmula com objetos que possuam significado geométrico explícitos. O pullback de $\epsilon(\mathcal{D})$, integrado em $L$, é igual a integral da curvatura de Lipschitz-Killing de $L$, ou o integrando do teorema de Gauss-Bonnet-Chern. Por mais ampla que seja essa classe de variedades Riemannianas, ou que as hipóteses se mantenham simples, como o fato de não exigir completude, questiona-se quando apenas os sinais das curvaturas seccionais seria o suficiente para controlar o sinal da curvatura de Lipschitz-Killing de $L$, como no teorema 10, e obter resultados análogos. No entanto, essa é uma pergunta em aberto,

Conjectura 1 (Hopf). Uma variedade com dimensão par e curvatura seccional positiva possui característica de Euler positiva.

É claro que, para variedades de dimensão dois, a resposta é trivial. Em termos de dimensões, o único caso não trivial com prova completa é o seguinte

Teorema 11 (Milnor, [50]). Seja M uma variedade Riemanniana compacta orientável de dimensão 4. Se suas curvaturas seccionais possuem sempre o mesmo sinal, $\chi(M) \geq O$. Se as curvaturas seccionais são sempre positivas ou sempre negativas, $\chi(M)>0$.

Como consta em [50], o resultado acima foi feito em comunicação oral de Milnor.

\section{Aplicações do teorema de Milnor}

Com efeito, tomando uma distribuição de dimensão 4, podemos remover a hipótese de curvatura pura. Logo,

Teorema 12 (Brito-Gonçalves, [7, 8]). Seja $\mathcal{D}^{4}$ uma distribuição numa variedade Riemanniana $M^{4+p}$. Seja L uma subvariedade fechada umbílica (totalmente geodésica) de $M$, com dimensão 4, e suponha que as curvaturas seccionais de $M$ sejam nãonegativas (positivas) quando restritas a L. Se $\mathcal{D}^{4}$ é tangente a L, então $\epsilon(\mathcal{D}) \neq 0$. 
Demonstração. A imersão $i: L \rightarrow M$ induz naturalmente $i^{*}: H^{4}(M, \mathbb{R}) \rightarrow H^{4}(L, \mathbb{R})$ e temos diretamente a integral $\int_{L} i^{*}(\epsilon(\mathcal{D}))$. Além disso, $L$ é subvariedade umbílica, donde vale

$$
\begin{aligned}
\epsilon(\mathcal{F}) & =\frac{1}{32 \pi^{2}} \sum_{\sigma \in \mathfrak{S}_{4}} \operatorname{sgn}(\sigma)\left(\Omega_{\sigma(1) \sigma(2)} \wedge \Omega_{\sigma(3) \sigma(4)}+2\|H\|^{2} \theta_{\sigma(1)} \wedge \theta_{\sigma(2)} \wedge \Omega_{\sigma(3) \sigma(4)}\right. \\
& \left.+\|H\|^{4} \theta_{\sigma(1)} \wedge \theta_{\sigma(2)} \wedge \theta_{\sigma(3)} \wedge \theta_{\sigma(4)}\right)
\end{aligned}
$$

Pelo teorema de Milnor, as curvaturas 4-seccionais, ie os coeficientes que acompanham os termos do tipo $\Omega_{\sigma(1) \sigma(2)} \wedge \Omega_{\sigma(3) \sigma(4)}$ na equação acima, são todas nãonegativas. Assim, podemos proceder como na demonstração do teorema 10. Portanto, $\epsilon(\mathcal{D}) \neq 0$ in $H^{4}(M, \mathbb{R})$.

Do mesmo modo, podemos derivar consequências similares àquelas obtidas através do teorema 10 . Se $\mathcal{F}$ é uma folheação por grandes círculos de $\mathbb{S}^{3}$, então a folheação produto em $M=\mathbb{S}^{3} \times \mathbb{S}^{3} \times \mathbb{S}^{3} \times \mathbb{S}^{3}$ também é geodésica, mas possui classe de Euler nula, pelo fato de $H^{4}(M)$ ser trivial. Isso mostra como a hipótese de termos curvatura positiva, no caso de uma folheação totalmente geodésica, não pode ser removida. 


\section{Folheações mensuráveis}

O teorema 12 aparece como alternativa à suposição sobre a diagonalização do operador de curvatura do ambiente folheado. Porém, em ambos os casos, teoremas 10 e 12, a existência de uma folha compacta é peça fundamental. Mesmo que possamos construir naturalmente folheações com folhas que gozam desta propriedade, via produtos Riemannianos por exemplo, a existência deste tipo de folha em folheações arbitrárias é um problema profundo na teoria. Isso se deve ao teorema de Sullivan e toda cultura que se criou em torno do problema de encontrar métrica que dá a folheação uma propriedade geométrica dada.

Esta seção tem como intuito principal substituir tal hipótese por duas suposições: a primeira é pedir que a variedade folheada seja fechada, e a segunda é ter uma medida transversal na folheação.

Consideremos a classe de Euler, $\epsilon(\mathcal{F}) \in H^{4}(M)$, agora de uma folheação de dimensão 4 em variedade Riemanniana fechada $M$. Se $\mathcal{F}$ admite uma folha compacta $L$, podemos considerar sua classe de homologia em $M$, dualizar via o teorema de Poincaré, e ter um elemento de $[L] \in H^{n-4}(M)$. Com efeito, a integral de $\epsilon(\mathcal{F}) \wedge[L]$ em $M$ é justamente a integral de uma função vezes o elemento de volume de $M$. O que faremos na sequência é a determinar uma classe em $H^{n-4}(M)$, via uma medida nas seções transversais, tal que a função integrada em $M$ tenha um sinal apropriado.

Definição 4 (Plante, [40]). Uma folheação mensurável é uma folheação munida de uma medida $\nu$ nas seções transversais, invariante perante o grupo de holonomia da folheação e é finita em conjuntos compactos.

A discussão anterior exibe o primeiro exemplo de folheação mensurável, quando existe uma folha compacta. Neste caso, $\nu$ é definida pela contagem de intersecções de um subconjunto de uma subvariedade transversal, com a folha compacta.

Em seguida, tratamos do exemplo de folheação mensurável que será útil para o caso análogo aos teoremas 10 e 12 .

A definição de uma folheação via cociclos de Haefliger é dada por uma cobertura por 
abertos $\left\{U_{i}\right\}$ de $M$ e submersões $f_{i}: U_{i} \rightarrow \mathbb{R}^{p}$, tais que existem únicos difeomorfismos

$$
\gamma_{i j}: f_{j}\left(U_{i} \cap U_{j}\right) \rightarrow f_{i}\left(U_{i} \cap U_{j}\right)
$$

satisfazendo $\gamma_{i j} \circ f_{j}=f_{i}$ em $U_{i} \cap U_{j}$ e a condição de cociclo, $\gamma_{i k} \gamma_{k j}=\gamma_{i j}$.

Definição 5. Uma folheação $\mathcal{F}$ de codimensão $p$ de uma variedade $M$ é dita ser uma $S L_{p^{-}}$ folheação quando os cociclos são tais que, para todos $i, j, \gamma_{i j}^{*} \omega_{\mathbb{R}^{p}}=\omega_{\mathbb{R}^{p}}$, onde $\omega_{\mathbb{R}^{p}}=d x_{1} \wedge$ $\cdots \wedge d x_{p}$

O fibrado normal de $\mathcal{F}$ pode ser definido via a sequencia exata $0 \rightarrow T \mathcal{F} \rightarrow T M \rightarrow \nu \mathcal{F} \rightarrow$ 0, e, neste caso, admite uma "forma de volume" peculiar. Defina uma $p$-forma no aberto $U_{i}$ por $\nu=f_{i}^{*} \omega_{\mathbb{R}^{p}}$. Na intersecção $U_{i} \cap U_{j}, f_{i}^{*} \omega_{\mathbb{R}^{p}}=\left(\gamma_{i j} \circ f_{j}\right)^{*} \omega_{\mathbb{R}^{p}}=f_{j}^{*} \omega_{\mathbb{R}^{p}}$, isto é, $\nu$ é globalmente definida em $M$. Já que $d \omega_{\mathbb{R}^{p}}=0$, e a diferencial exterior comuta com o pullback, $\nu$ também é fechada.

Ademais, sejam $X, Y$ seções suaves de $M$, e denote por $X^{\top}$ e $X^{\perp}$ as projeções tangencial e normal de $X$, respectivamente. Assim, defina

$$
A(X, Y)=\left(\nabla_{Y^{\perp}} X^{\perp}\right)^{\top}
$$

Seguindo o referencial adaptado $\left\{e_{i}, e_{\alpha}\right\}$ em $\mathrm{M}$, definimos $A_{\alpha \beta}^{i}=\left\langle A\left(e_{\alpha}, e_{\beta}\right), e_{i}\right\rangle$. Dado um ponto $x \in M$, a aplicação $h$ é a segunda forma fundamental da folha que passa por $x$. Para $A$, temos resultados semelhantes, mas envolvendo a geometria transversal de $\mathcal{F}$. De acordo com [27], definição 1.2.2 na página $7, \mathcal{F}$ é uma folheação Riemanniana quando $A$ é antissimétrica. E essa propriedade caracteriza folheações Riemannianas. Além disso, $\left[X^{\perp}, Y^{\perp}\right]^{\top}=A(Y, X)-$ $A(X, Y)$. Ou seja, $\nu \mathcal{F}$ é integrável se e somente se $A$ é simétrico. Análogo ao campo de curvatura média, considere

$$
H^{\perp}=\operatorname{tr} A=\sum_{i, \alpha} A_{\alpha \alpha}^{i} e_{i}
$$

Assim, temos caracterizações de SL-folheações, de acordo com o teorema 6.32, página 71 de [49], 
Teorema 13 (Tondeur, [49]). Seja $\mathcal{F}$ uma folheação de codimensão p numa variedade Riemanniana M. São equivalentes

(i) $\mathcal{F}$ é $S L_{p}$-folheação.

(ii) $\nu$ é uma p-forma global e fechada.

(iii) $H^{\perp} \equiv 0$.

(iv) $\mathcal{L}(X) \nu=0$, para todo $X \in \Gamma \mathcal{F}$, onde $\mathcal{L}$ denota a derivada de Lie.

Consequentemente, folheações Riemannianas são exemplos de SL-folheações. Além disso, a caracterização via $H^{\perp} \equiv 0$ mostra que fluxos associados aos campos solenoidais também formam uma classe de exemplos de SL-folheações.

\section{Número de Euler de uma folheação}

Uma medida transversal em uma folheação mensurável $(\mathcal{F}, \nu)$ de codimensão $p$ define um elemento em $H^{p}(M)$. Seguindo [40], seja $\left\{U_{i}\right\}$ uma cobertura local de $M$ por vizinhanças distinguidas, isto é, vizinhanças difeomorfas ao produto de discos abertos $D^{p} \times D^{n-p}$, na qual $\mathcal{F}$ corresponde a folheação trivial quando restrita a $U_{i}$. Sejam $\lambda_{i}$ uma partição da unidade subordinada a cobertura local, $\psi_{i}: D^{p} \times D^{n-p} \rightarrow U_{i}$ coordenadas locais distinguidas, e $\eta$ uma $(n-p)$-forma fechada em $M$, com suporte compacto. O elemento de $H^{p}(M)$ definido por $\nu$, que por abuso de notação denotamos também por $\nu$, é dado por

$$
\nu(\eta)=\sum_{i} \pm \int_{D^{p}}\left(\int_{\{x\} \times D^{n-p}} \psi_{i}^{*}\left(\lambda_{i} \eta\right)\right) d\left(\psi_{i}^{*} \nu\right)(x)
$$

onde o sinal \pm aparece pois depende da orientação induzida em $D^{p}$ via a folheação; $\psi_{i}^{*} \nu$ denota a medida induzida em $D^{p}$ de $\nu$ via $\psi_{i}$. Além disso, pode ser mostrado que $\nu(\eta)$ independe da escolha da cobertura nem da partição subordinada. Para mais detalhes, veja [40] e [43]

Em [20], define-se um número associado a uma folheação mensurável $(\mathcal{F}, \nu)$ de dimensão 
par, chamado número de Euler de $\mathcal{F}$,

$$
\chi(\mathcal{F}, \nu)=\int_{M} \epsilon(\mathcal{F}) \wedge \nu
$$

Connes discute como $\chi(\mathcal{F}, \nu)$ pode ser interpretado como uma média das características de Euler das folhas de uma folheação mensurável, exibindo $\chi(\mathcal{F}, \nu)$ em termos das somas dos graus dos zeros de campos em cada folha, como no caso clássico do teorema de Poincaré-Hopf, bem como a construção de $\epsilon(\mathcal{F})$ como fizemos anteriormente, via o Pfaffiano das formas de curvatura de cada folha. Porém, na tentativa de escrever $\chi(\mathcal{F}, \nu)$ em termos das somas (com sinal) dos números de Betti, Connes encontra um problema. Neste caso, o autor exibe uma maneira alternativa de definir números de Betti para folheações mensuráveis. Entretanto, mesmo que a abordagem do autor para os novos números de Betti fuja do escopo deste trabalho, uma das consequências é o seguinte resultado,

Teorema 14 (Connes, [20]). Seja $\mathcal{F}$ uma folheação nensurável por superfícies em uma variedade fechada M. Se o conjundo das $S^{2}$-folhas possui medida $\mu$ nula, então $\chi(\mathcal{F}, \nu) \leq 0$, onde $\mu$ é uma medida global em $M$, definida por $\nu$ e pela forma de área das folhas de $\mathcal{F}$.

Parafraseando Connes, o teorema acima pode ser interpretado geometricamente como: se existe curvatura positiva suficiente em uma folha genérica, então esta folha deve ser fechada e logo uma esfera.

\section{Resultados}

Finalmente, obtemos uma combinação dos resultados exibidos até então; a demonstração de Milnor para a caso de dimensão quatro da conjectura de Hopf e a definição de Connes do número de Euler de uma folheação.

Teorema 15 (Brito-Gonçalves, [7, 8]). Seja $\mathcal{F}$ uma SL-folheação de dimensão 4 em uma variedade Riemanniana fechada $M^{4+p}$. Se as curvaturas seccionais das folhas tem sempre o mesmo sinal, então $\chi(\mathcal{F}, \nu)=\int_{M} \epsilon(\mathcal{F}) \wedge \nu \geq 0$. 
Demonstração. Pela definição de $\epsilon(\mathcal{F})$,

$$
\begin{aligned}
\int_{M} \epsilon(\mathcal{F}) \wedge \nu & =\frac{1}{32 \pi^{2}} \sum_{\sigma \in \mathfrak{S}_{4}} \operatorname{sgn}(\sigma) \int_{M} \Omega_{\sigma(1) \sigma(2)}^{L} \wedge \Omega_{\sigma(3) \sigma(4)}^{L} \wedge \nu \\
& =\frac{1}{32 \pi^{2}}\left(\begin{array}{c}
p+4 \\
4
\end{array}\right) \int_{M} \gamma_{4}^{L} .
\end{aligned}
$$

donde o resultado é obtido pelo teorema de Milnor.

Como o resultado do Connes, temos uma intuição geométrica do teorema anterior: fixe uma $S L$-folheação, dimensão 4, de uma variedade Riemanniana fechada. Se o conjunto das folhas, tais que suas curvaturas seccionais mudam de sinal possui medida nula, então a característica de Euler desta folheação deve ser positiva. 


\section{Capítulo 4}

\section{Sobre invariantes conformes e construção de exem-}

plos

Este capítulo enumera as propriedades invariantes conformes presentes nas hipóteses essenciais dos resultados do capítulo anterior, bem como uma demonstração de que a pureza de curvatura, característica do ambiente folheado no teorema 10 e dos exemplos daquele capítulo, é invariante perante mudança conforme na métrica. Consequentemente, exemplos de folheações umbílicas, especificamente em variedades com forma pura de curvatura, tornam-se imediatos, sempre que tais ambientes admitam folheações totalmente geodésicas.

A seção 4.2 exibe exemplos encontrados na literatura. Podemos observar como a construção presente em [30] pode ser estendida para fibrações por grandes círculos da esfera, em qualquer dimensão. Desse modo, combinado ao teorema 2, e aos resultados de [25], vemos o quão abundantes são folheações umbílicas em esferas Euclidianas.

Com hipóteses sobre a topologia das folhas e sobre a 1-forma de curvatura associada, estudamos a existência de uma métrica para a qual uma folheação umbílica é totalmente geodésica, respondendo parcialmente uma pergunta feita em [15]. Ademais, recentemente, Lytchak e Wilking [32] reuniram as peças restantes para a conclusão da classificação de folheações Riemannianas em variedades homeomorfas a esferas. Utilizamos esta classificação, em particular o levantamento histórico feito pelos autores, para concluir que folheações umbílicas (tais que a 1-forma de curvatura seja fechada) e totalmente geodésicas em esferas Euclidianas não possuem fibrado normal integrável. 


\section{Invariantes conformes}

Definição 6 (Métrica conforme). Seja $(M,\langle\cdot, \cdot\rangle)$ uma variedade Riemanniana e seja $f$ : $M \rightarrow \mathbb{R}$ uma função suave. A métrica $\langle\cdot, \cdot\rangle^{\prime}=e^{2 f}\langle\cdot, \cdot\rangle$ é dita ser conforme à métrica inicial de $M$.

Baseados na seção 2.1 do capítulo 2, exibiremos as relações entre as novas conexão, formas de conexão e curvatura $\nabla^{\prime}, \omega_{A B}^{\prime}$ e $\Omega_{A B}^{\prime}$, respectivamente, com os recíprocos dados inicialmente pela métrica $\langle\cdot, \cdot\rangle$. Primeiramente, lembremos da fórmula de Koszul

$$
2\left\langle\nabla_{X} Y, Z\right\rangle=X\langle Y, Z\rangle+Y\langle X, Z\rangle-Z\langle X, Y\rangle+\langle[X, Y], Z\rangle-\langle[X, Z], Y\rangle-\langle[Y, Z], X\rangle
$$

Denotando por $\nabla f$ o gradiente de $f$,

$$
\begin{aligned}
2\left\langle\nabla_{X}^{\prime} Y, Z\right\rangle^{\prime} & =X\langle Y, Z\rangle^{\prime}+Y\langle X, Z\rangle^{\prime}-Z\langle X, Y\rangle^{\prime}+\langle[X, Y], Z\rangle^{\prime}-\langle[X, Z], Y\rangle^{\prime}-\langle[Y, Z], X\rangle^{\prime} \\
& =2 e^{2 f} X(f)\langle Y, Z\rangle+2 e^{2 f} Y(f)\langle X, Z\rangle-2 e^{2 f} Z(f)\langle X, Y\rangle \\
& +e^{2 f} X\langle Y, Z\rangle+e^{2 f} Y\langle X, Z\rangle-e^{2 f} Z\langle X, Y\rangle \\
& +e^{2 f}\langle[X, Y], Z\rangle-e^{2 f}\langle[X, Z], Y\rangle-e^{2 f}\langle[Y, Z], X\rangle \\
& =2 e^{2 f} X(f)\langle Y, Z\rangle+2 e^{2 f} Y(f)\langle X, Z\rangle-2 e^{2 f}\langle\nabla f, Z\rangle\langle X, Y\rangle \\
& +2 e^{2 f}\left\langle\nabla_{X} Y, Z\right\rangle \\
& =2\left(X(f)\langle Y, Z\rangle^{\prime}+Y(f)\langle X, Z\rangle^{\prime}-\langle X, Y\rangle\langle\nabla f, Z\rangle^{\prime}+\left\langle\nabla_{X} Y, Z\right\rangle^{\prime}\right)
\end{aligned}
$$

Logo,

$$
\nabla_{X}^{\prime} Y=\nabla_{X} Y+X(f) Y+Y(f) X-\langle X, Y\rangle \nabla f
$$

O referencial ortonormal e coreferencial para a nova métrica são dados por $\left\{e_{A}^{\prime}=e^{-f} e_{A}\right\}$ 
e $\left\{\omega_{A}^{\prime}=e^{f} \omega_{A}\right\}$, respectivamente. Então,

$$
\begin{aligned}
\omega_{A B}^{\prime}(X) & =\left\langle\nabla_{X}^{\prime} e_{A}^{\prime}, e_{B}^{\prime}\right\rangle^{\prime}=e^{2 f}\left\langle\nabla_{X}^{\prime}\left(e^{-f} e_{A}\right), e^{-f} e_{B}\right\rangle \\
& =e^{f}\left\langle\nabla_{X}\left(e^{-f} e_{A}\right)+X(f)\left(e^{-f} e_{A}\right)+\left(e^{-f} e_{A}\right)(f) X-\left\langle X, e^{-f} e_{A}\right\rangle \nabla f, e_{B}\right\rangle \\
& =\left\langle-X(f) e_{A}+\nabla_{X} e_{A}+X(f) e_{A}+e_{A}(f) X-\left\langle X, e_{A}\right\rangle \nabla f, e_{B}\right\rangle \\
& =\left\langle\nabla_{X} e_{A}+e_{A}(f) X-\left\langle X, e_{A}\right\rangle \nabla f, e_{B}\right\rangle \\
& =\omega_{A B}(X)+f_{A}\left\langle X, e_{B}\right\rangle-f_{B}\left\langle X, e_{A}\right\rangle \\
& =\omega_{A B}(X)+f_{A} \omega_{B}(X)-f_{B} \omega_{A}(X)
\end{aligned}
$$

donde

$$
\omega_{A B}^{\prime}=\omega_{A B}+f_{A} \omega_{B}-f_{B} \omega_{A},
$$

onde $f_{A}=e_{A}(f)=d f\left(e_{A}\right)=\left\langle\nabla f, e_{A}\right\rangle$.

O cálculo das novas formas de curvatura é dado da seguinte maneira

$$
\begin{aligned}
\Omega_{A B}^{\prime} & =-d \omega_{A B}^{\prime}+\sum_{C} \omega_{A C}^{\prime} \wedge \omega_{C B}^{\prime} \\
& =-d\left(\omega_{A B}+f_{A} \omega_{B}-f_{B} \omega_{A}\right)+\sum_{C}\left(\omega_{A C}+f_{A} \omega_{C}-f_{C} \omega_{A}\right) \wedge\left(\omega_{C B}+f_{C} \omega_{B}-f_{B} \omega_{C}\right) \\
& =-d \omega_{A B}-d f_{A} \wedge \omega_{B}-f_{A} d \omega_{B}+d f_{B} \wedge \omega_{A}+f_{B} d \omega_{A} \\
& +\sum_{C} \omega_{A C} \wedge \omega_{C B}+\sum_{C} f_{C} \omega_{A C} \wedge \omega_{B}-\sum_{C} f_{B} \omega_{A C} \wedge \omega_{C}+\sum_{C} f_{A} \omega_{C} \wedge \omega_{C B} \\
& +\sum_{C} f_{A} f_{C} \omega_{C} \wedge \omega_{B}-\sum_{C} f_{C} \omega_{A} \wedge \omega_{C B}-\sum_{C} f_{C}^{2} \omega_{A} \wedge \omega_{B}+\sum_{C} f_{C} f_{B} \omega_{A} \wedge \omega_{C} \\
& =\Omega_{A B}-d f_{A} \wedge \omega_{B}+d f_{B} \wedge \omega_{A}+\sum_{C} f_{C} \omega_{A C} \wedge \omega_{B} \\
& +\sum_{C} f_{A} f_{C} \omega_{C} \wedge \omega_{B}-\sum_{C} f_{C} \omega_{A} \wedge \omega_{C B}-\sum_{C} f_{C}^{2} \omega_{A} \wedge \omega_{B}+\sum_{C} f_{C} f_{B} \omega_{A} \wedge \omega_{C}
\end{aligned}
$$

Assuma que $M$ possua forma pura de curvatura. Logo, suponha que o referencial $\left\{e_{A}\right\}$ seja tal que $\Omega_{A B}=c_{A B} \omega_{A} \wedge \omega_{B}$. Enunciamos o seguinte resultado,

Teorema 16. Pureza de curvatura é um invariante conforme.

Demonstração. A ideia da prova é usar a equação que relaciona $\Omega_{A B}$ e $\Omega_{A B}^{\prime}$ e verificar que o referencial $\left\{e_{A}^{\prime}\right\}$ é tal que $\Omega_{A B}^{\prime}=c_{A B}^{\prime} \omega_{A}^{\prime} \wedge \omega_{B}^{\prime}$, exibindo as funções $c_{A B}^{\prime}$ em termos de $c_{A B}$ 
e das componentes do gradiente de $f$.

$$
\begin{aligned}
\Omega_{A B}^{\prime}\left(e_{A}, e_{B}\right) & =\left(\Omega_{A B}-d f_{A} \wedge \omega_{B}+d f_{B} \wedge \omega_{A}+\sum_{C} f_{C} \omega_{A C} \wedge \omega_{B}+\sum_{C} f_{A} f_{C} \omega_{C} \wedge \omega_{B}\right. \\
& \left.-\sum_{C} f_{C} \omega_{A} \wedge \omega_{C B}-\sum_{C} f_{C}^{2} \omega_{A} \wedge \omega_{B}+\sum_{C} f_{C} f_{B} \omega_{A} \wedge \omega_{C}\right)\left(e_{A}, e_{B}\right) \\
& =c_{A B}-d f_{A}\left(e_{A}\right)+d f_{B}\left(e_{B}\right)+f_{A}^{2}+f_{B}^{2} \\
& +\sum_{C} f_{C} \omega_{A C}\left(e_{A}\right)+f_{C} \omega_{B C}\left(e_{B}\right)-f_{C}^{2}
\end{aligned}
$$

Portanto, (compare com o teorema 1.159, página 58 de [4])

$$
c_{A B}^{\prime}=e^{2 f}\left(c_{A B}-d f_{A}\left(e_{A}\right)+d f_{B}\left(e_{B}\right)+f_{A}^{2}+f_{B}^{2}\right)+e^{2 f} \sum_{C}\left(f_{C} \omega_{A C}\left(e_{A}\right)+f_{C} \omega_{B C}\left(e_{B}\right)-f_{C}^{2}\right)
$$

Podemos mostrar que subvariedades umbílicas são invariantes perante mudança conforme na métrica. De fato, com a notação definida na seção 2.1, temos

$$
\left(h_{i j}^{\alpha}\right)^{\prime}=e^{2 f} \theta_{i \alpha}^{\prime}\left(e_{j}^{\prime}\right)=e^{f}\left(\theta_{i \alpha}\left(e_{j}\right)+f_{i} \omega_{\alpha}\left(e_{j}\right)-f_{\alpha} \omega_{i}\left(e_{j}\right)\right)=e^{f}\left(h_{i j}^{\alpha}-f_{\alpha} \delta_{i j}\right),
$$

e como umbilicidade implica $h_{i j}^{\alpha}=\lambda_{\alpha} \delta_{i j}$, tal propriedade permanece com a mudança na métrica.

Exemplos de folheações umbílicas em variedades com forma pura de curvatura, aquelas as quais aparecem nos teoremas 10 e 12, podem ser obtidas a partir do seguinte algoritmo. Tome uma variedade $M$ munida de uma métrica com forma pura de curvatura, tal que $M$ possua uma folheação totalmente geodésica; por exemplo, uma hipersuperfície isoparamétrica numa forma espacial, com a folheação dada pela distribuição principal de uma curvatura principal (para verificar que esta distribuição constitui uma folheação totalmente geodésica, basta usar a equação 2.7), ou uma folheação da esfera Euclidiana por grandes círculos. Seja $f: M \rightarrow \mathbb{R}$ uma função suave, constante ao longo das folhas da folheação. Então, uma mudança conforme por um fator $e^{2 f}$ torna a folheação umbílica, e preserva a pureza de curvatura.

Finalmente, usamos os procedimentos anteriores para exibir uma folheação umbílica, que 
não é totalmente geodésica, com todas as folhas compactas e com classe de Euler trivial. O objetivo é mostrar como a hipótese da nãonegatividade das curvaturas seccionais do ambiente é fundamental para a conclusão dos teoremas do capítulo anterior.

Seja $\mathcal{F}$ uma fibração de $\mathbb{S}^{3}$ por grandes círculos. A folheação produto $\mathcal{F} \times \mathcal{F}$ é totalmente geodésica em $\mathbb{S}^{3} \times \mathbb{S}^{3}$. Se $f$ é uma função em $\mathbb{S}^{3} \times \mathbb{S}^{3}$, suave, constante ao longo das folhas de $\mathcal{F} \times \mathcal{F}$, então a métrica $e^{2 f}\langle\cdot, \cdot\rangle$, onde $\langle\cdot, \cdot\rangle$ é a métrica produto canônica de $\mathbb{S}^{3} \times \mathbb{S}^{3}$, é tal que $\mathcal{F} \times \mathcal{F}$ é umbílica. As folhas, do tipo $\mathbb{S}^{1} \times \mathbb{S}^{1}$, são umbílicas e compactas. Porém, pelo fato de $H^{2}\left(\mathbb{S}^{3} \times \mathbb{S}^{3}\right)$ ser trivial, $\epsilon(\mathcal{F} \times \mathcal{F})=0$ (independente da métrica). Isso também se deduz diretamente pela equação (3.4). De fato, a classe de Euler se escreve como

$$
\epsilon(\mathcal{F} \times \mathcal{F})=-\frac{1}{2 \pi}\left(\gamma_{2}+\|H\|^{2}\right) \theta_{1} \wedge \theta_{2}
$$

onde $\gamma_{2}$ é a curvatura seccional dos planos tangentes a $\mathcal{F} \times \mathcal{F}$, e $H$ é o campo de curvatura média da folheação. Pela demonstração do teorema 4.1, $\gamma_{2}=-e^{2 f} \sum_{\alpha} f_{\alpha}^{2}$. Finalmente, a equação 4.3 nos permite calcular a curvatura média, $H=-e^{f} \sum_{\alpha} f_{\alpha} e_{\alpha}$. 


\section{Construção de exemplos na esfera Euclidiana}

Em [30], os autores constroem folheações umbílicas, em $\mathbb{S}^{3}$, a partir de fibrações de Hopf, via perturbação nos planos de $\mathbb{R}^{4}$ que interceptados com a esfera determinam as fibras da fibração. Uma vez que o procedimento apresentado independe da natureza transversal da fibração, nem das dimensões das fibras, apresentamos a seguir uma construção para qualquer fibração de $\mathbb{S}^{2 n+1}$ por grandes esferas.

Seja $F^{2 k+1}$ uma fibração de $\mathbb{S}^{2 n+1}$ por grandes círculos. Tomamos $2 k+1$ pois existe uma restrição nas possíveis dimensões de uma fibração de $\mathbb{S}^{2 n+1}$ por grandes círculos; para mais detalhes, [25]. Se $\widetilde{G}_{2 k+2}\left(\mathbb{R}^{2 n+2}\right)$ é o conjunto dos $(2 k+2)$-planos de $\mathbb{R}^{2 n+2}$, defina

$$
\widetilde{G}_{2 k+2}^{F}\left(\mathbb{R}^{2 n+2}\right)=\left\{P \in \widetilde{G}_{2 k+2}\left(\mathbb{R}^{2 n+2}\right): P \cap \mathbb{S}^{2 n+1} \text { é fibra de } F\right\}
$$

Fixe $x \in \mathbb{S}^{2 n+1}$ e seja $\epsilon>0$ dado, suficientemente pequeno. Considere $\widetilde{G}_{2 k+2}^{F}\left(\mathbb{R}^{2 n+2}, \epsilon x\right)$ conjunto dos planos $\epsilon x+P$ paralelos a $P$, contendo o ponto $\epsilon x$. Por definição, existe único $P_{x}$ em $F$, tal que $x \in P_{x}$. Já que $P_{x}$ contém a origem de $\mathbb{R}^{2 n+2}$, então $\epsilon x \in P_{x}$, donde os planos $P_{x}$ e $\epsilon x+P_{x}$ coincidem. Desse modo, $\left(\epsilon x+P_{x}\right) \cap \mathbb{S}^{2 n+1}$ é um grande círculo de $\mathbb{S}^{2 n+1}$.

Sejam $P$ e $P^{\prime}$ elementos de $\widetilde{G}_{2 k+2}^{F}\left(\mathbb{R}^{2 n+2}\right)$.

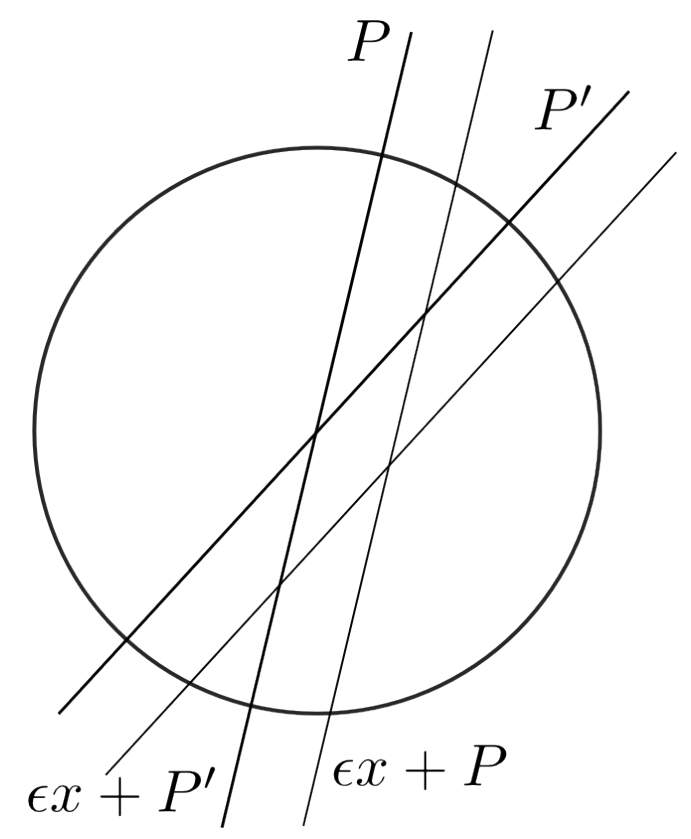

Figura 4.1: Perturbação dos planos em $\mathbb{R}^{2 n+2}$ 
Proposição 2. Se $\left(P \cap P^{\prime}\right) \cap \mathbb{S}^{2 n+1}=\emptyset$, então $(\epsilon x+P) \cap\left(\epsilon x+P^{\prime}\right) \cap \mathbb{S}^{2 n+1}=\emptyset$.

Demonstração. De fato, se y é um ponto da intersecção $(\epsilon x+P) \cap\left(\epsilon x+P^{\prime}\right) \cap \mathbb{S}^{2 n+1}$, então $y-\epsilon x \in\left(P \cap P^{\prime}\right)$. Logo, $\frac{y-\epsilon x}{\|y-\epsilon x\|} \in\left(P \cap P^{\prime}\right) \cap \mathbb{S}^{2 n+1}$.

Proposição 3. Dado $y \in \mathbb{S}^{2 n+1}$, existe único $P$ de modo que $y \in \epsilon x+P$.

Demonstração. Seja $z=\frac{y-\epsilon x}{\|y-\epsilon x\|} \in \mathbb{S}^{2 n+1}$. Logo, existe $P_{z} \in \widetilde{G}_{2 k+2}^{F}\left(\mathbb{R}^{4}\right)$ que contém $z$, donde $\epsilon x+P_{z}$ contém o elemento $y$. A unicidade é consequência imediata da proposição anterior.

De acordo com o raciocínio anterior, para o $x \in \mathbb{S}^{2 n+1}$ fixado inicialmente, $\left(\epsilon x+P_{x}\right) \cap \mathbb{S}^{2 n+1}$ é um grande círculo de $\mathbb{S}^{2 n+1}$. Agora, para qualquer outro elemento $P$ de $\widetilde{G}_{2 k+2}^{F}\left(\mathbb{R}^{2 n+2}\right)$, temos que $(\epsilon x+P) \cap \mathbb{S}^{2 n+1}$ é um pequeno círculo, isto é, uma subvariedade umbílica. Assim, a partição $\left\{(\epsilon x+P) \cap \mathbb{S}^{2 n+1}: P \in \widetilde{G}_{2 k+2}^{F}\left(\mathbb{R}^{2 n+2}\right)\right\}$ é uma folheação umbílica de $\mathbb{S}^{2 n+1}$, com uma folha totalmente geodésica.

Portanto, dada uma fibração de $\mathbb{S}^{2 n+1}$ por grandes círculos, cada fibra determina uma folheação umbílica de $\mathbb{S}^{2 n+1}$. 


\section{Folheações umbílicas com fibrado normal integrável}

Baseado na seção 4.1, gostaríamos de saber se existem folheações umbílicas $\mathcal{F}^{m}$ na esfera Euclidiana $\mathbb{S}^{n}$, tal que $\mathcal{F}$ tenha fibrado normal integrável.

Se as folhas de $\mathcal{F}$ são subvariedades completas, então tais folhas são esferas de dimensão $m$, em $\mathbb{S}^{n}$, obtidas via intersecção da esfera com subespaços afim e não-lineares (que não contém a origem) do espaço Euclidiano $\mathbb{R}^{n+1}$; ver [3], página 25. Desse modo, essa folheação possui todas as folhas compactas. Pelo teorema 10, $m$ não pode ser par, donde $m=2 k+1$, para algum inteiro $k \geq 0$. Enfatizamos que $\mathcal{F}$ pode possuir folhas totalmente geodésicas, ou seja, provindas da intersecção com subespaços lineares. Neste caso, analogamente, as deduções anteriores referentes a dimensão e a compacidade continuam válidas.

Por outro lado, folheações regulares, i.e. com todas as folhas de mesma dimensão, são encontradas apenas em esferas de dimensão ímpar. Isso se deve ao seguinte raciocínio; a característica de Euler de $\mathbb{S}^{2 n}$ é igual a dois. Se $\mathbb{S}^{2 n}$ admitisse folheação regular $\mathcal{F}$, então a classe de Euler da esfera poderia ser escrita como $\epsilon\left(T \mathbb{S}^{2 n}\right)=\epsilon(T \mathcal{F}) \epsilon(\nu \mathcal{F})$, e ambas $\epsilon(T \mathcal{F})$ e $\epsilon(\nu \mathcal{F})$ são nulas pela cohomologia de $\mathbb{S}^{2 n}$, uma contradição. Dessa maneira, consideraremos folheações em $\mathbb{S}^{2 n+1}$.

Uma folha umbílica $L$ significa, em termos das notações que introduzimos nas preliminares, que $\theta_{i \alpha}=\lambda_{\alpha} \theta_{i}$, para $\lambda_{\alpha}: \mathbb{S}^{2 n+1} \rightarrow \mathbb{R}$, e assumimos que esta função seja suave em $\mathbb{S}^{2 n+1}$. Em termos da segunda forma fundamental, $\lambda_{\alpha}=h_{i i}^{\alpha}$, para qualquer $1 \leq \ldots i \cdots \leq 2 k+1$.

Lema 3. O campo H, a curvatura média da folheação umbilica, é constante ao longo das folhas de $\mathcal{F}$.

Demonstração. Num espaço de curvatura constante, os coeficientes da derivada covariante de $h_{i j}^{\alpha}$ satisfazem a relação $\sum_{j, k} h_{i j k}^{\alpha} \theta_{i} \wedge \theta_{k}=0$, para todo $i$ e todo $\alpha$. Para verificar esta equação, basta aplicar o lema 2.5. Como uma folha $L$ é umbílica, $h_{i j}^{\alpha}=\lambda_{\alpha} \delta_{i j}$. Então $\sum_{k} h_{i i k}^{\alpha} \theta_{i} \wedge \theta_{k}=0$. Por outro lado, o lema de Cartan implica que $h_{i i k}^{\alpha}=h_{i k i}^{\alpha}$, donde $h_{i i k}^{\alpha}=0$ para todo $k \neq i$. Porém, temos $0=\sum_{k} h_{i i k}^{\alpha} \theta_{i} \wedge \theta_{k}\left(e_{i}, e_{k}\right)=\sum_{k \neq i} h_{i i k}^{\alpha}+h_{i i i}$. Logo, $\lambda_{\alpha}$ é constante ao longo de $L$, donde $H$ também o é. 
No lema acima, a hipótese de estarmos num ambiente de curvatura constante é usada para verificar que as componentes da derivada covariante das entradas da segunda forma fundamental satisfazem uma equação do tipo Codazzi.

Defina $\kappa(\cdot)=\langle H, \cdot\rangle$, a 1 -forma de curvatura de $\mathcal{F}$. Então, para um ambiente Riemanniano qualquer,

Teorema 17. Seja $\mathcal{F}$ uma folheação umbilica numa variedade Riemanniana $M$, na qual a 1-forma de curvatura é exata em $M$. Então $M$ admite uma métrica na qual $\mathcal{F}$ é totalmente geodésica.

Demonstração. Uma vez que $\kappa$ é exata, $H=\nabla F$, para alguma função $F: M \rightarrow \mathbb{R}$. Se $\langle\cdot, \cdot\rangle$ denota a métrica inicial em $M$, defina $\langle\cdot, \cdot\rangle^{\prime}:=e^{2 F}\langle\cdot, \cdot\rangle$. Pela equação (4.3) e o fato que $F_{\alpha}=\left\langle\nabla F, e_{\alpha}\right\rangle=\lambda_{\alpha}, \mathcal{F}$ é totalmente geodésica em $\left(M,\langle\cdot, \cdot\rangle^{\prime}\right)$.

Para campos $X, Y$ na esfera, a diferencial exterior de $\kappa$ em $\mathbb{S}^{2 n+1}$ pode ser escrita como

$$
d \kappa(X, Y)=X(\kappa(Y))-Y(\kappa(X))-\kappa([X, Y])
$$

O lema anterior mostra que, na esfera Euclidiana, $H$ é constante ao longo das folhas. Logo, $d \kappa\left(X_{1}, X_{2}\right)=0$ quando $X_{1}$ e $X_{2}$ são tangentes a folheação. Assuma que o fibrado normal de $\mathcal{F}$ seja integrável, e denote tal folheação por $\mathcal{G}$. Desse modo, se $\xi_{1}$ e $\xi_{2}$ são tangentes a $\mathcal{G}$, então $\left[\xi_{1}, \xi_{2}\right]$ também é. Assim, $d \kappa\left(\xi_{1}, \xi_{2}\right)=0$.

A partir de agora, assuma que $d \kappa(X, \xi)=0$, para $X \in \mathfrak{X F}$ e $\xi \in \mathfrak{X G}$. Isso significa que $\kappa$ é fechada, donde exata pois $H^{1}\left(\mathbb{S}^{2 n+1}\right)=0$. Consequentemente, pelo teorema anterior $\mathcal{G}$ é uma folheação Riemanniana de $\left(\mathbb{S}^{2 n+1},\langle\cdot, \cdot\rangle^{\prime}\right)$, pois, seu fibrado normal, agora $\mathcal{F}$, é totalmente geodésica.

Uma vez que $\left(\mathbb{S}^{2 n+1},\langle\cdot, \cdot\rangle^{\prime}\right)$ é homeomorfa a esfera Euclidiana, olhemos para como se comportam as folheações Riemannianas de esferas, ou variedades homeomorfas. A ideia central é verificar que estas folheações acontecem em determinadas dimensões, todas ímpares. Como a folheação inicial é de dimensão ímpar, $2 k+1$, em uma esfera de dimensão $2 n+1$, a folheação $\mathcal{G}$ é necessariamente de dimensão par, o que é uma contradição. Assim, podemos 
concluir o seguinte:

Teorema 18. A esfera Euclidiana não admite folheações umbilicas com fibrado normal integrável, tais que $d \kappa(X, \xi)=0$, para $X \in \Gamma \mathcal{F}$ e $\xi \in \Gamma \mathcal{G}$.

O raciocínio da dedução das possíveis dimensões das folheações Riemannianas em esferas foi feito em [32], e trata de coletar peças necessárias na literatura. Por questões de completude, reproduzimos a seguir as ideias principais levantadas pelos autores.

Dada uma folheação Riemanniana de uma variedade homeomorfa a esfera, Ghys em [23] mostrou que as folhas genéricas são fechadas, a menos de serem de dimensão um, que neste caso são dadas por um fluxo isométrico perante possivelmente uma nova métrica no ambiente. Além disso, as folhas são esferas de homotopia racional. Em [28], Haefliger notou que qualquer folheação Riemanniana em uma variedade completa $M$, com folhas fechadas, pode-se encontrar uma nova variedade ambiente, homotópica a $M$, que seja o espaço total de um fibrado cujo as fibras são homotópicas as folhas da folheação Riemanniana inicial. No caso de $M$ ser uma esfera, as fibras (ou topologicamente as folhas) são contráteis em M. Porém, Spanier e Whitehead já haviam observado em [46] que, no caso de um fibrado deste tipo, as fibras constituem um $H$-espaço, isto é, as fibras possuem uma identidade de grupo, mas que é homotópica a aplicação de identidade da fibra. Agora, basta olhar para a literatura e verificar que, em [13], Browder classificou as variedades fechadas que são $H$ espaços e esferas de homotopia racional. São homotopicamente equivalentes as seguintes: $\mathbb{S}^{1}$, $\mathbb{S}^{3}, \mathbb{R P}^{3}, \mathbb{S}^{7}$ e $\mathbb{R P}^{7}$

Com isso, as possíveis dimensões de uma folheação Riemanniana em uma esfera são $1,3,7$. 


\section{Capítulo 5}

\section{Hipersuperfícies Euclidianas: campos de vetores, folheações e o grau da aplicação normal}

O objetivo principal deste capítulo é relacionar campos unitários em uma hipersuperfície fechada Euclidiana e a geometria da hipersuperfície, com o grau da aplicação normal de Gauss, via fórmulas integrais. Exibiremos uma lista de invariantes topológicos e enunciaremos uma aplicação a teoria de folheações totalmente geodésicas nestes espaços. Além disso, mostraremos como objetos dependentes exclusivamente da geometria da hipersuperfície se relacionam com o grau da aplicação normal.

Seja $M^{n+1}(c)$ uma variedade Riemanniana fechada com curvatura seccional constante $c$, e seja $\mathcal{F}$ uma folheação transversalmente orientada em $M(c)$, de codimensão um. Brito et al, em [10], mostraram que a média das curvaturas médias de ordem superior, i.e. as integrais das funções simétricas dos autovalores da segunda forma fundamental das folhas, não dependem da folheação.

$$
\int_{M} \sigma_{k}= \begin{cases}c^{k / 2}\left(\begin{array}{c}
n / 2 \\
k / 2
\end{array}\right) \operatorname{vol}(M), & \text { se } k \text { e } n \text { são pares, } \\
0, & \text { se } k \text { ou } n \text { é ímpar. }\end{cases}
$$

Desde então, diversas fórmulas integrais foram encontradas, por meio de transformações de Newton e para codimensões maiores; vide [1] e [42] para mais detalhes. As integrais foram calculadas em [10] através de uma aplicação definida por Milnor em [34], e também utilizada em [2] para calcular a integral acima para o caso $\sigma_{n / 2}=K$, curvatura de Gauss- 
Kronecker das folhas; para o caso de curvatura positiva, seja $\varphi_{t}: \mathbb{S}^{n+1} \rightarrow \mathbb{S}^{n+1}\left(\sqrt{1+t^{2}}\right)$, $\varphi_{t}(x)=x+t N(x)$, onde $N$ e o campo unitário, normal a $\mathcal{F}$. Assim, as integrais surgem por uma comparação de coeficientes em $t$, que aparecem através do determinante da matriz Jacobiana de $\varphi_{t}$, e do volume de $\mathbb{S}^{n+1}\left(\sqrt{1+t^{2}}\right)$.

Todos os casos conhecidos, [1], [10], [42] e as referências neles contidas, as fórmulas integrais são independentes da folheação, mas ainda dependem da geometria do ambiente folheado.

Na primeira seção, definimos uma aplicação similar a $\varphi_{t}$, mas com uma hipersuperfície Euclidiana como domínio. Essa nova aplicação depende do fibrado normal de um campo unitário, da segunda forma fundamental e da aplicação normal da hipersuperfície. Com efeito, obtemos fórmulas integrais, as quais se resumem ao caso positivo supracitado, e que dependem da topologia da hipersuperfície através do grau da aplicação normal.

\section{A aplicação $\varphi_{t}^{\vec{v}}$}

Seja $M^{n+1}$ uma hipersuperfície imersa, orientada e fechada do espaço Euclidiano $\mathbb{R}^{n+2}$, com $\chi(M)=0$, e seja $\vec{v}: M \rightarrow T M$ um campo vetorial suave em $M$. Fixe uma orientação de $M$ de modo que $\nu: M^{n+1} \rightarrow \mathbb{S}^{n+1}$ seja bem definida, $\nu(x)=N(x)$, onde $N$ é campo unitário, normal a $M$.

Para um número real $t>0$, defina $\varphi_{t}^{\vec{v}}: M^{n+1} \rightarrow \mathbb{R}^{n+2}$, por $\varphi_{t}^{\vec{v}}(x)=\nu(x)+t \vec{v}(x)$. Ambos $\nu(x)$ e $\vec{v}(x)$ são unitários em $\mathbb{R}^{n+2}$, para qualquer $x \in M$; $\operatorname{logo},\left\|\varphi_{t}^{\vec{v}}(x)\right\|^{2}=1+t^{2}$, o que significa que a imagem de $\varphi_{t}^{\vec{v}}$ está contida na esfera $\mathbb{S}^{n+1}\left(\sqrt{1+t^{2}}\right)$ de raio $\sqrt{1+t^{2}}$. Por outro lado, $\nu$ e $\vec{v}$ são aplicações suaves, e somar também é uma aplicação suave em $\mathbb{R}^{n+2}$, donde $\varphi_{t}^{\vec{v}}$ é suave.

A fórmula do grau, ver Guillermin-Pollack página 188, diz que para qualquer aplicação suave $f: X \rightarrow Y$ entre variedades com mesma dimensão, $\operatorname{dim}(X)=\operatorname{dim}(Y)=k$, e para $\omega$ uma $k$-forma em $Y$, vale

$$
\int_{X} f^{*} \omega=\operatorname{deg}(f) \int_{Y} \omega
$$

No caso de $\varphi_{t}^{\vec{v}}: M^{n+1} \rightarrow \mathbb{S}^{n+1}\left(\sqrt{1+t^{2}}\right)$, tome $\omega$ como sendo a forma de volume de $\mathbb{S}^{n+1}\left(\sqrt{1+t^{2}}\right)$, e vol$\left(\mathbb{S}^{n+1}\right)$ como o volume da esfera unitária. Então, o lado direito da equação 
(5.2) torna-se

$$
\begin{aligned}
\operatorname{deg}\left(\varphi_{t}^{\vec{v}}\right) \int_{\mathbb{S}^{n+1}\left(\sqrt{1+t^{2}}\right)} \omega & =\operatorname{deg}\left(\varphi_{t}^{\vec{v}}\right) \operatorname{vol}\left(\mathbb{S}^{n+1}\left(\sqrt{1+t^{2}}\right)\right)=\operatorname{deg}\left(\varphi_{t}^{\vec{v}}\right) \operatorname{vol}\left(\mathbb{S}^{n+1}\right)\left(\sqrt{1+t^{2}}\right)^{n+1} \\
& =\operatorname{deg}\left(\varphi_{t}^{\vec{v}}\right) \operatorname{vol}\left(\mathbb{S}^{n+1}\right)\left(\sqrt{1+t^{2}}\right)\left(\sqrt{1+t^{2}}\right)^{n}
\end{aligned}
$$

Para o lado esquerdo de (5.2), existe uma relação entre o pullback de $\omega$ via $\varphi_{t}^{\vec{v}}$ e a forma de volume de $M, \omega_{M}$. Neste caso,

$$
\int_{M}\left(\varphi_{t}^{\vec{v}}\right)^{*} \omega=\int_{M} \operatorname{det}\left(d \varphi_{t}^{\vec{v}}\right) \omega_{M}
$$

\section{Invariantes topológicos}

Estamos interessados em calcular o determinante de $d\left(\varphi_{t}^{\vec{v}}\right)_{x}: T_{x} M^{n+1} \rightarrow T_{\varphi_{t}^{\vec{v}}(x)} \mathbb{S}^{n+1}\left(\sqrt{1+t^{2}}\right)$.

Como o determinante é invariante perante transformações de similaridade, podemos escolher quaisquer bases ortonormais de $T_{x} M^{n+1}$ e $T_{\varphi_{t}^{\vec{v}}(x)} \mathbb{S}^{n+1}\left(\sqrt{1+t^{2}}\right)$ para calcular a matriz $d\left(\varphi_{t}^{\vec{v}}\right)$. Para $T_{x} M^{n+1}$, tome o seguinte conjunto adaptado de vetores ortonormais,

$$
\left\{e_{1}, \ldots, e_{n}, \vec{v}\right\}
$$

Note que $\varphi_{t}^{\vec{v}}(x) \perp e_{i}$, para todo $1 \leq i \leq n$, e que $\varphi_{t}^{\vec{v}}(x)$ pode ser escolhido como vetor normal a $\mathbb{S}^{n+1}\left(\sqrt{1+t^{2}}\right)$, já que é ponto desta esfera. Desse modo, podemos completar o conjunto $\left\{e_{1}, \ldots, e_{n}\right\}$ com um vetor no qual é simultaneamente perpendicular a todos $e_{i}, 1 \leq i \leq n$, e $\varphi_{t}^{\vec{v}}(x)$. Logo,

$$
\left\{e_{1}, \ldots, e_{n}, u:=\frac{\vec{v}}{\sqrt{1+t^{2}}}-t \frac{N}{\sqrt{1+t^{2}}}\right\}
$$

é uma base ortonormal para $T_{\varphi_{t}^{\vec{v}}(x)} \mathbb{S}^{n+1}\left(\sqrt{1+t^{2}}\right)$.

Se $S=S_{N}: T M \rightarrow T M$ é o operador forma de $M$, então $d \nu(\cdot)=S(\cdot)=\left(D_{(\cdot)} N\right)^{\top}$, onde $(\cdot)^{\top}$ denota a projeção em $T M$, e $D$ a conexão Euclidiana. Se $\nabla$ é a conexão de Levi-Civita induzida de $M$, então

$$
d \vec{v}(\cdot)=D_{(\cdot)} \vec{v}=\nabla_{(\cdot)} \vec{v}+\left(D_{(\cdot)} \vec{v}\right)^{\perp}=\nabla_{(\cdot)} \vec{v}+\left\langle D_{(\cdot)} \vec{v}, N\right\rangle N=\nabla_{(\cdot)} \vec{v}-\langle\vec{v}, S(\cdot)\rangle N
$$


Com respeito a notação supracitada, a aceleração $\nabla_{\vec{v}} \vec{v}$ possui as seguintes componentes: $v_{i}:=\left\langle\nabla_{\vec{v}} \vec{v}, e_{i}\right\rangle$ e $\langle S(\vec{v}), \vec{v}\rangle=h_{n+1 n+1}$. Evidentemente, $v_{n+1}=\left\langle\nabla_{\vec{v}} \vec{v}, \vec{v}\right\rangle=0$. Ademais, definimos $a_{i j}:=\left\langle\nabla_{e_{i}} \vec{v}, e_{j}\right\rangle$. A matriz $\left(a_{i j}\right)$ localmente descreve o comportamento do fibrado normal de $\vec{v}$; e.g. $\left(a_{i j}\right)$ é simétrica se e só se o fibrado normal é integrável a uma folheação de codimensão um de $M$. Da equação (5.7), $d \vec{v}\left(e_{i}\right)=\nabla_{e_{i}} \vec{v}-\left\langle\vec{v}, S\left(e_{i}\right)\right\rangle N=\nabla_{e_{i}} \vec{v}-h_{n+1}{ }_{i} N$, onde $h_{A B}$ são as entradas da segunda forma fundamental de $M$, e $d \vec{v}(\vec{v})=\nabla_{\vec{v}} \vec{v}-\langle\vec{v}, S(\vec{v})\rangle N=$ $\nabla_{\vec{v}} \vec{v}-h_{n+1 n+1} N$.

Temos

$$
\begin{aligned}
\left\langle d \varphi_{t}^{\vec{v}}\left(e_{i}\right), e_{j}\right\rangle & =\left\langle d \nu\left(e_{i}\right)+t d \vec{v}\left(e_{i}\right), e_{j}\right\rangle=\left\langle S\left(e_{i}\right)+t \nabla_{e_{i}} \vec{v}-t h_{n+1}{ }_{i} N, e_{j}\right\rangle=h_{i j}+t a_{i j} \\
\left\langle d \varphi_{t}^{\vec{v}}\left(e_{i}\right), u\right\rangle & =\left\langle S\left(e_{i}\right)+t \nabla_{e_{i}} \vec{v}-t h_{n+1} N, \frac{\vec{v}}{\sqrt{1+t^{2}}}-t \frac{N}{\sqrt{1+t^{2}}}\right\rangle \\
& =\left\langle S\left(e_{i}\right), \frac{\vec{v}}{\sqrt{1+t^{2}}}\right\rangle-\left\langle S\left(e_{i}\right), t \frac{N}{\sqrt{1+t^{2}}}\right\rangle+\left\langle t \nabla_{e_{i}} \vec{v}, \frac{\vec{v}}{\sqrt{1+t^{2}}}\right\rangle \\
& -\left\langle t \nabla_{e_{i}} \vec{v}, t \frac{N}{\sqrt{1+t^{2}}}\right\rangle-\left\langle t h_{n+1} N, \frac{\vec{v}}{\sqrt{1+t^{2}}}\right\rangle+\left\langle t h_{n+1} N, t \frac{N}{\sqrt{1+t^{2}}}\right\rangle \\
& =\frac{1}{\sqrt{1+t^{2}}}\left\langle S\left(e_{i}\right), \vec{v}\right\rangle+\frac{t}{\sqrt{1+t^{2}}}\left\langle\nabla_{e_{i}} \vec{v}, \vec{v}\right\rangle+\frac{t^{2}}{\sqrt{1+t^{2}}} h_{n+1 i}\langle N, N\rangle \\
& =\frac{1}{\sqrt{1+t^{2}}} h_{i n+1}+\frac{t^{2}}{\sqrt{1+t^{2}}} h_{n+1} i \\
& =\sqrt{1+t^{2}} h_{n+1 i}
\end{aligned}
$$

$$
\begin{aligned}
\left\langle d \varphi_{t}^{\vec{v}}(\vec{v}), e_{i}\right\rangle & =\left\langle d \nu(\vec{v})+t d \vec{v}(\vec{v}), e_{i}\right\rangle=\left\langle S(\vec{v})+t \nabla_{\vec{v}} \vec{v}-t v_{N} N, e_{i}\right\rangle=h_{n+1}+t v_{i} \\
\left\langle d \varphi_{t}^{\vec{v}}(\vec{v}), u\right\rangle & =\left\langle d \varphi_{t}^{\vec{v}}(\vec{v}), \frac{\vec{v}}{\sqrt{1+t^{2}}}-t \frac{N}{\sqrt{1+t^{2}}}\right\rangle \\
& =\left\langle S(\vec{v})+t \nabla_{\vec{v}} \vec{v}-t v_{N} N, \frac{\vec{v}}{\sqrt{1+t^{2}}}-t \frac{N}{\sqrt{1+t^{2}}}\right\rangle \\
& =\frac{1}{\sqrt{1+t^{2}}} v_{N}+\frac{t^{2}}{\sqrt{1+t^{2}}} v_{N} \\
& =\sqrt{1+t^{2}} v_{N}
\end{aligned}
$$


Então

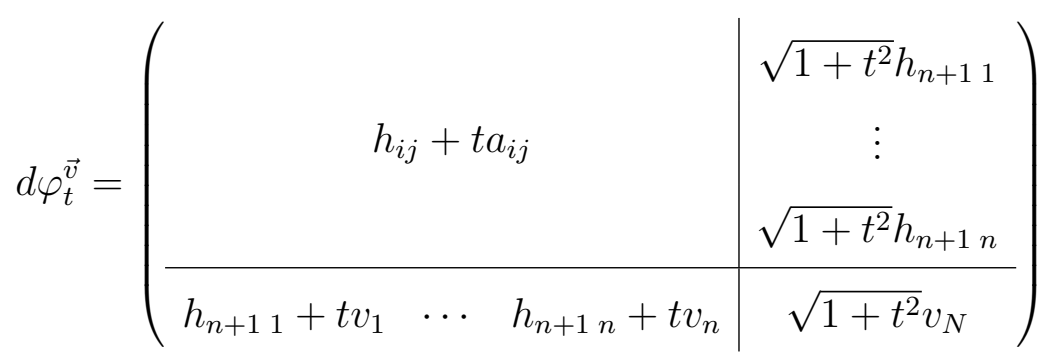

Definindo vetores coluna

$$
V_{j}=\left(a_{1 j}, \ldots, a_{n j}, v_{j}\right), \quad H_{j}=\left(h_{1 j}, \ldots, h_{n+1 j}\right), \quad H_{n+1}=\left(h_{n+11}, \ldots, h_{n+1 n}, v_{N}\right),
$$

podemos reescrever a matriz acima como

$$
d \varphi_{t}^{\vec{v}}=\left(\begin{array}{llll}
H_{1}+t V_{1} & \cdots & H_{n}+t V_{n} & \sqrt{1+t^{2}} H_{n+1}
\end{array}\right) .
$$

O determinante é linear com respeito a soma e multiplicação por escalares nos vetores coluna, logo, a equação (5.8) simplifica os cálculos no que diz respeito a uma fórmula explícita para $\operatorname{det}\left(d \varphi_{t}^{\vec{v}}\right)$, escrita em termos da segunda forma fundamental de $M$ e das componentes que dependem do fibrado normal de $\vec{v}$. Assim,

$$
\begin{aligned}
& \operatorname{det}\left(d \varphi_{t}^{\vec{v}}\right)=\sqrt{1+t^{2}} \sum_{k=0}^{n} \eta_{k} t^{k}, \quad \text { onde } \\
& \eta_{0}=\operatorname{det}\left(h_{A B}\right), 1 \leq A, B \leq n+1 \\
& \eta_{1}=\sum_{i} \operatorname{det}\left(\begin{array}{lllll}
H_{1} & \cdots & V_{i} & \cdots & H_{n+1}
\end{array}\right) \\
& \eta_{2}=\sum_{i<j} \operatorname{det}\left(\begin{array}{lllllll}
H_{1} & \cdots & V_{i} & \cdots & V_{j} & \cdots & H_{n+1}
\end{array}\right) \\
& \text { ! } \\
& \eta_{n}=\operatorname{det}\left(\begin{array}{llll}
V_{1} & \cdots & V_{n} & H_{n+1}
\end{array}\right)
\end{aligned}
$$

Pelas equações (5.3) e (5.4),

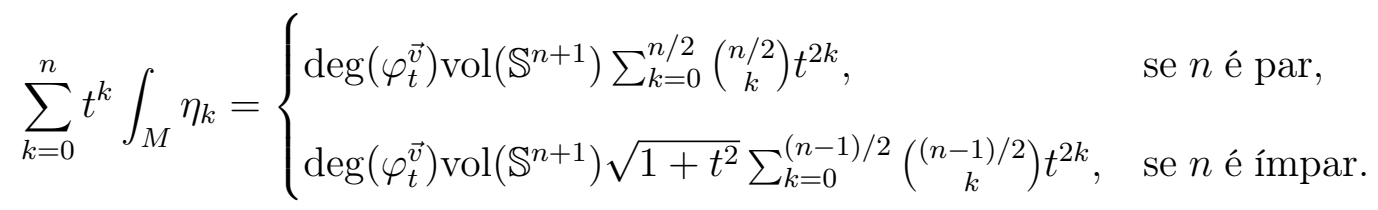


Observamos que, quando $n$ é par ambos os lados da equação anterior são polinômios na variável $t$, e as potências do lado direito são potências pares. Isso implica que os coeficientes multiplicando potências ímpares no lado esquerdo são todos nulos. Quando $n$ é ímpar, o termo $\sqrt{1+t^{2}}$ mostra que todos os coeficientes no lado esquerdo são zero. Por outro lado, para $t$ suficientemente pequeno, temos que os graus das aplicações $\varphi_{t}^{\vec{v}}$ e $\nu$ coincidem, $\operatorname{deg}\left(\varphi_{t}^{\vec{v}}\right)=\operatorname{deg}(\nu)$. Então concluímos que

$$
\int_{M} \eta_{k}= \begin{cases}\operatorname{deg}(\nu)\left(\begin{array}{l}
n / 2 \\
k / 2
\end{array}\right) \operatorname{vol}\left(\mathbb{S}^{n+1}\right), & \text { se } k \text { e } n \text { são pares } \\
0, & \text { se } k \text { ou } n \text { é ímpar. }\end{cases}
$$

No lado esquerdo da equação, temos as funções $\eta_{k}$, que dependem do fibrado normal de $\vec{v}$, através da matriz $a_{i j}$, e da segunda forma fundamental de $M$, pela matriz $h_{A B}$. Ou seja, tais funções são de cunho geométrico. O lado direito apresenta, além das constantes combinatórias e do volume da esfera, o grau da aplicação normal, invariante topológico, que depende da classe de homotopia da imersão de $M$ no Euclidiano.

\section{Possíveis valores para o grau da aplicação normal}

Ponha $\operatorname{deg}(\nu)=d$. Em [33], foi provado que

Teorema 19 (Milnor, [33]). Fixe n impar,

(a) Se $M$ pode ser imersa em $\mathbb{R}^{n+1}$ com grau d, então pode ser imersa com qualquer graud d', tal que d'é congruente a d modulo 2.

(b) Se $M$ pode ser imersa em $\mathbb{R}^{n+1}$ com grau zero, então $M$ é paralelizável.

(c) Assuma que $M$ não é paralelizável. Se $M$ pode ser imersa em $\mathbb{R}^{n+1}$, então pode ser imersa com grau impar arbitrário, mas não pode ser imersa com grau par.

O primeiro item nos mostra quão abundante são os possíveis valores de $d$. No entanto, para mergulhos, existem restrições para o grau que dependem dos números de Betti da hipersuperfície,

Teorema 20 (Milnor, [33]). Para qualquer mergulho de $M^{n}$ em $\mathbb{R}^{n+1}$ o grau d da aplicação 
normal satisfaz

$$
2 d \equiv \beta(M) \quad \bmod 2, \quad 2|d| \leq \beta(M),
$$

onde $\beta(M)=\beta_{0}(M)+\beta_{1}(M)+\cdots+\beta_{n}(M)$ denota a soma dos números de Betti da variedade M. Ademais, se $M$ é orientada, então $2-\frac{1}{2} \beta(M) \leq d \leq \frac{1}{2} \beta(M)$.

Para um número $d$ aparecer como o grau de uma imersão, o anel $H^{*}(M)$ deve se quebrar em soma direta de duas subálgebras, e $d$ deve aparecer como a característica de Euler de uma delas. Para mais detalhes, e.g. provas dos teoremas e exemplos, ver [33]. Veja também [45] para o caso dos possíveis valores do grau para imersões de esferas. 


\section{Consequências para geometria de folheações}

Seja $\mathcal{F}^{2 n}$ uma folheação transversalmente orientada de codimensão um de uma hipersuperfície fechada, orientada e imersa $M^{2 n+1}$ de $\mathbb{R}^{2 n+2}$. Tome $\vec{v}$ como campo unitário normal às folhas. Neste caso, em cada ponto de $M$, a matriz $\left(a_{i j}\right)$ representa a segunda forma fundamental da folha passando por este ponto, e, portanto, é simétrica. Logo,

$$
\eta_{2 n}=\operatorname{det}\left(\begin{array}{ccc|c} 
& & & h_{2 n+11} \\
& & & \vdots \\
& a_{i j} & \\
& & & h_{2 n+12 n} \\
\hline v_{1} & \cdots & v_{2 n} & h_{2 n+12 n+1}
\end{array}\right) \text {. }
$$

Se $\eta_{2 n}=0$ (ou para qualquer $1 \leq k \leq n, \eta_{2 k}=0$ ) então (5.9) implica que $\operatorname{deg}(\nu)=0$.

Teorema 21 (Brito-Gonçalves, [9]). Se $M^{2 n+1}$ admite um campo unitário, tal que a matriz associada a segunda forma fundamental do fibrado normal possui posto menor ou igual a $2(n-1)$, então $\operatorname{deg}(\nu)=0$, onde $\nu: M^{2 n+1} \rightarrow \mathbb{S}^{2 n+1}$ é a aplicação normal de Gauss.

O teorema anterior combinado ao item (b) de 19 implica que $M^{2 n+1}$ é paralelizável. Por outro lado, uma folheação totalmente geodésica de $M$ claramente satisfaz a hipótese sobre o posto da matriz $\left(a_{i j}\right)$. Logo,

Corolário 4. Se $M$ admite uma folheação totalmente geodésica de codimensão um, então o grau da aplicação normal de $M$ é zero.

Considerando o teorema 19, podemos concluir que entre todas imersões de uma dada hipersuperfície fechada, e todos os possíveis valores que $\operatorname{deg}(\nu)$ pode assumir, folheações satisfazendo $\operatorname{rank}\left(a_{i j}\right) \leq 2(n-1)$ (e portanto as totalmente geodésicas) podem ocorrer apenas quando $\operatorname{deg}(\nu)=0$. No espírito dos itens $(b)$ e $(c)$ do teorema 19, Smale, em [45], provou o seguinte resultado,

Teorema 22 (Smale, [45]). Existe imersão da esfera $\mathbb{S}^{n+1}$ em $\mathbb{R}^{n+2} \operatorname{com} \operatorname{deg}(\nu)=0$ se $\mathbb{S}^{n+1}$ for paralelizável. 
Consequentemente, as únicas possíveis esferas que poderiam admitir uma folheação de codimensão um com a hipótese acima sobre o posto da matriz $a_{i j}$ são $\mathbb{S}^{3}$ e $\mathbb{S}^{7}$, imersas com grau zero. Porém, $\mathbb{S}^{3}$ não admite esse tipo de folheação, para qualquer métrica Riemanniana: a suposição sobre o posto implica total geodesibilidade em $\mathbb{S}^{3}$. De fato, pelo teorema de Novikov, qualquer folheação de $\mathbb{S}^{3}$ possui uma componente de Reeb, i.e., um toro sólido, com seu interior folheado por folhas difeomorfas a $\mathbb{R}^{2}$. Pelo teorema de Sullivan, o bordo deste toro, compacto, que também é uma folha, não admite curva transversal fechada.

Corolário 5. Seja $f: \mathbb{S}^{2 n+1} \rightarrow \mathbb{R}^{2 n+2}$ uma imersão, $n \neq 3$, e seja $\mathcal{F}$ uma folheação suave de codimensão um de $f\left(\mathbb{S}^{2 n+1}\right)$. Então a nulidade da segunda forma fundamental das folhas é menor que 2 .

Assim, surge a seguinte questão: existe imersão de $\mathbb{S}^{7}$ no espaço Euclidiano, que pode ser folheada por uma folheação com $\operatorname{rank}\left(a_{i j}\right) \leq 2(n-1)$ ? 


\section{O campo $\vec{v}$ é uma direção principal}

Assuma que $\vec{v}$ seja um autovetor do operador de Weingarten de $M$, com $S(\vec{v})=\lambda \vec{v}$ e $\lambda$ tenha multiplicidade um em todo $M$. Isso significa que $\lambda \neq \lambda_{k}$, para todas as outras curvaturas principais $\lambda_{k}, 1 \leq k \leq n$. Neste caso, as funções $\eta_{k}$ dependem exclusivamente da geometria extrínseca de $M$; são funções das curvaturas principais e das derivadas destas em outras direções principais.

Na sequência, provaremos o seguinte resultado

Teorema 23. Em termos da notação acima,

$$
\int_{M} \lambda \sum_{k} \frac{d \lambda_{k}(\vec{v})}{\lambda_{k}-\lambda} \lambda_{1} \cdots \widehat{\lambda_{k}} \cdots \lambda_{n}=0
$$

Note que as curvaturas $\lambda_{k} \neq \lambda$ podem ter multiplicidades distintas, e/ou serem nulas.

A ideia da demonstração é simples: mostrar que o integrando é a função $\eta_{1}$ e aplicar 5.9. De fato, por definição e pela Proposição 1,

$$
a_{k k}=\left\langle\nabla_{e_{k}} \vec{v}, e_{k}\right\rangle=\frac{d \lambda_{k}(\vec{v})}{\lambda_{k}-\lambda}, \quad v_{k}=\frac{d \lambda\left(e_{k}\right)}{\lambda_{k}-\lambda}
$$

Já que $\vec{v}$ é uma direção principal, podemos tomar o referencial $\left\{e_{1}, \ldots, e_{n}, \vec{v}\right\}$ de modo que $h_{A B}=0$, para $A \neq B$. Assim, os vetores coluna $H_{A}$ e $V_{j}$ que compõem $\eta_{1}$ são dados por

$$
H_{A}=\left(0, \cdots \lambda_{A}, \cdots 0\right), \quad V_{j}=\left(a_{1 j}, \ldots \frac{d \lambda_{k}(\vec{v})}{\lambda_{k}-\lambda}, \ldots a_{n j}, \frac{d \lambda\left(e_{j}\right)}{\lambda_{j}-\lambda}\right)
$$

Consequentemente,

$$
\begin{aligned}
\eta_{1} & =\sum_{i} \operatorname{det}\left(\begin{array}{ccccc}
H_{1} & \cdots & V_{i} & \cdots & H_{n+1}
\end{array}\right) \\
& =\lambda \sum_{k} \frac{d \lambda_{k}(\vec{v})}{\lambda_{k}-\lambda} \lambda_{1} \cdots \widehat{\lambda_{k}} \cdots \lambda_{n}
\end{aligned}
$$

Vejamos agora o caso de dimensão 3 e a relação com o grau da aplicação normal de Gauss. Suponha que $M$ possua 3 curvaturas principais distintas, $\mu, \eta$ e $\lambda$, com $S(\vec{v})=\lambda \vec{v}$. O fibrado 
normal de $\vec{v}$ constitui uma distribuição em $M$, possui dimensão dois, não necessariamente integrável e é gerado pelas direções principais $e_{1}$ e $e_{2}$. Denote por $(\cdot)^{\perp}$ a projeção neste espaço. Por meio de transporte paralelo, podemos assumir que, pontualmente, para toda campo $X$ em $M,\left(\nabla_{X} e_{i}\right)^{\perp}=0$. Com a compatibilidade da conexão com a métrica de $M$, traduzida pela equação 2.6, e pela Proposição 1 temos

$$
\begin{aligned}
a_{12} & =\left\langle\nabla_{e_{1}} \vec{v}, e_{2}\right\rangle=\left\langle\left[e_{1}, \vec{v}\right], e_{2}\right\rangle=\left\langle\sum_{k} C_{13 k} e_{k}, e_{2}\right\rangle=C_{132} \\
& =\Gamma_{32}^{1}-\Gamma_{12}^{3}=\frac{h_{123}(\mu-\lambda)}{(\lambda-\eta)(\mu-\eta)}
\end{aligned}
$$

analogamente,

$$
a_{21}=\frac{h_{123}(\eta-\lambda)}{(\lambda-\mu)(\eta-\mu)}
$$

Portanto,

$$
\operatorname{deg}(\nu) \operatorname{vol}\left(\mathbb{S}^{3}\right)=\int_{M} \lambda\left(\frac{d \mu(\vec{v}) d \eta(\vec{v})}{(\mu-\lambda)(\eta-\lambda)}+\frac{h_{123}^{2}}{(\mu-\eta)^{2}}\right)
$$

Finalmente, espera-se que as relações acima sejam úteis para o estudo de problemas de pinching de curvatura, como no caso da conjectura de Chern, [44]. 
50 HIPERSUPERFÍCIES EUCLIDIANAS: CAMPOS DE VETORES, FOLHEAÇÕES E O GRAU DA APLICAÇÃO NORMAL 


\section{Referências Bibliográficas}

[1] Krzysztof Andrzejewski and Paweł G Walczak. The newton transformation and new integral formulae for foliated manifolds. Annals of Global Analysis and Geometry, 37(2):103-111, 2010. 39, 40

[2] Daniel Asimov. Average gaussian curvature of leaves of foliations. Bulletin of the American Mathematical Society, 84(1):131-133, 1978. 3, 39

[3] Jurgen Berndt, Sergio Console, and Carlos Enrique Olmos. Submanifolds and holonomy, volume 21. CRC Press, 2016. 36

[4] Arthur L Besse. Einstein manifolds. Springer Science \& Business Media, 2007. 12, 32

[5] Raoul Bott. Lectures on characteristic classes and foliations. In Lectures on algebraic and differential topology, pages 1-94. Springer, 1972. 4

[6] Fabiano Brito. A remark on minimal foliations of codimension two. Tohoku Mathematical Journal, Second Series, 36(3):341-350, 1984. 4, 16

[7] Fabiano Brito and Icaro Gonçalves. Corrigendum to "The Euler class of an umbilic foliation"[CR Acad. Aci. Paris, Ser. I 354 (6)(2016) 614-618]. Comptes Rendus Mathematique, 2016. 22, 27

[8] Fabiano Brito and Icaro Gonçalves. The Euler class of an umbilic foliation. Comptes Rendus Mathematique, 354(6):614-618, 2016. 4, 5, 18, 22, 27

[9] Fabiano Brito and Icaro Gonçalves. Degree of the gauss map and curvature integrals for closed hypersurfaces. preprint, arXiv:1609.04670[math.DG] 2016. 4, 46

[10] Fabiano Brito, Rémi Langevin, and Harold Rosenberg. Intégrales de courbure sur des variétés feuilletées. Journal of differential geometry, 16(1):19-50, 1981. 3, 39, 40

[11] Fabiano G B Brito. Une obstruction géométrique à l'existence de feuilletages de codimension 1 totalement géodésiques. Journal of Differential Geometry, 16(4):675-684, 1981. 2,12

[12] Fabiano G B Brito and Paweł G Walczak. Totally geodesic foliations with integrable normal bundles. Bol. Soc. Bras. Mat, 17:41-46, 1986. 2

[13] William Browder. Higher torsion in h-spaces. Transactions of the American Mathematical Society, 108(2):353-375, 1963. 38

[14] Marco Brunella and Étienne Ghys. Umbilical foliations and transversely holomorphic flows. J. Differential Geom, 41(1):1-19, 1995. 2 
[15] Grant Cairns. Totally umbilic riemannian foliations. Michigan Math. J, 37:145-159, 1990. 29

[16] Grant Cairns and Étienne Ghys. Totally geodesic foliations on 4-manifolds. Journal of Differential Geometry, 23(3):241-254, 1986. 3

[17] Yves Carrière. Flots riemanniens et feuilletages géodésibles de codimension un. Universite des sciences et techniques de Lille 1, 1981. 1

[18] Thomas E Cecil and Patrick J Ryan. Geometry of Hypersurfaces. Springer, 2015. 10

[19] Shiing-shen Chern. A simple intrinsic proof of the gauss-bonnet formula for closed riemannian manifolds. Annals of mathematics, pages 747-752, 1944. 17

[20] Alain Connes. A survey of foliations and operator algebras. In Proc. Symp. Pure Math, volume 38, pages 521-628, 1982. 26, 27

[21] Marcos Dajczer, Vladimir Rovenski, and Ruy Tojeiro. Euclidean hypersurfaces with a totally geodesic foliation of codimension one. Geometriae Dedicata, 176(1):215-224, 2015. 3

[22] Étienne Ghys. Classification des feuilletages totalement géodésiques de codimension un. Commentarii Mathematici Helvetici, 58(1):543-572, 1983. 1

[23] Étienne Ghys. Feuilletages riemanniens sur les variétés simplement connexes. In Annales de l'institut Fourier, volume 34, pages 203-223, 1984. 38

[24] Herman Gluck and Frank W Warner. Great circle fibrations of the three-sphere. Duke Math. J, 50(1):107-132, 1983. 2

[25] Herman Gluck, Frank W Warner, and C T Yang. Division algebras, fibrations of spheres by great spheres and the topological determination of space by the gross behavior of its geodesics. Duke Math. J, 50:1041-1076, 1983. 2, 29, 34

[26] André Gomes. The mean curvature of a transversely orientable foliation. Results in Mathematics, 46(1-2):31-36, 2004. 2

[27] Detlef Gromoll and Gerard Walschap. Metric foliations and curvature, volume 268. Springer Science \& Business Media, 2009. 25

[28] André Haefliger. Groupö̈des d'holonomie et classifiants. Université de Genève-Section de mathématiques, 1982. 38

[29] David L Johnson and A M Naveira. A topological obstruction to the geodesibility of a foliation of odd dimension. Geometriae Dedicata, 11(3):347-352, 1981. 4, 16

[30] Aso Kazutoshi and Shinsuke Yorozu. A generalization of Clairaut's theorem and umbilical foliations. Nihonkai Mathematical Journal, 2(2):139-153, 1991. 29, 34

[31] Shoshichi Kobayashi and Katsumi Nomizu. Foundations of differential geometry, vol. II, Interscience, New York, 1969, volume 38. 1996. 16, 17 
[32] Alexander Lytchak and Burkhard Wilking. Riemannian foliations of spheres. Geom. Topol., 20:1257-1274, 2016. 29, 38

[33] John Milnor. On the immersion ofn-manifolds in $(\mathrm{n}+1)$-space. Commentarii Mathematici Helvetici, 30(1):275-284, 1956. 44, 45

[34] John Milnor. Analytic proofs of the"hairy ball theorem"and the brouwer fixed point theorem. The American Mathematical Monthly, 85(7):521-524, 1978. 39

[35] Maria H Noronha. Variedades com operador de curvatura puro. Universidade Estadual de Campinas, 1983. 13

[36] Tominosuke Ôtsuki. Minimal hypersurfaces in a riemannian manifold of constant curvature. American Journal of Mathematics, 92(1):145-173, 1970. 10

[37] Richard S Palais and Chuu-Lian Terng. Critical point theory and submanifold geometry. Springer, 1988. 7

[38] Joel S Pasternack. Foliations and compact lie group actions. Commentarii Mathematici Helvetici, 46(1):467-477, 1971. 4

[39] Peter Petersen. Riemannian geometry, volume 171. Springer Science \& Business Media, 2006. 12,13

[40] Joseph F Plante. Foliations with measure preserving holonomy. Annals of Mathematics, pages $327-361,1975.24,26$

[41] Georges Reeb. Sur la courbure moyenne des variétés intégrales d'une équation de Pfaff $\omega=0$. Comptes Rendus Mathematique, 231(2):101-102, 1950. 3

[42] Vladimir Rovenski and Paweł Walczak. Topics in extrinsic geometry of codimension-one foliations. Springer Science \& Business Media, 2011. 39, 40

[43] David Ruelle and Dennis Sullivan. Currents, flows and diffeomorphisms. Topology, 14(4):319-327, 1975. 26

[44] M Scherfner, S Weiss, and ST Yau. A review of the chern conjecture for isoparametric hypersurfaces in spheres. ALM, 21:1-13. 49

[45] Stephen Smale. The classification of immersions of spheres in euclidean spaces. Annals of mathematics, pages 327-344, 1959. 45, 46

[46] Edwin H Spanier and John H C Whitehead. On fibre spaces in which the fibre is contractible. Commentarii Mathematici Helvetici, 29(1):1-8, 1955. 38

[47] Dennis Sullivan. A homological characterization of foliations consisting of minimal surfaces. Commentarii Mathematici Helvetici, 54(1):218-223, 1979. 1

[48] John A Thorpe. Sectional curvatures and characteristic classes. Annals of Mathematics, pages $429-443,1964.11,12$

[49] Philippe Tondeur. Foliations on Riemannian manifolds. Springer Science \& Business Media, 2012. 25, 26 
[50] Shiing-shen Von Chern. On curvature and characteristic classes of a riemann manifold. In Abhandlungen aus dem Mathematischen Seminar der Universität Hamburg, volume 20, pages 117-126. Springer, 1955. 22

[51] Gerard Walschap. Umbilic foliations and curvature. Illinois Journal of Mathematics, 41(1):122-128, 1997. 2 